\title{
Functional evolution of quantum cylindrical waves
}

\author{
Demian H.J. Cho ${ }^{1, *}$ and Madhavan Varadarajan ${ }^{1, \dagger}$ \\ 1 Raman Research Institute, Bangalore 560 080, India
}

(Dated: October 7, 2018)

\begin{abstract}
Kuchař showed that the quantum dynamics of (1 polarization) cylindrical wave solutions to vacuum general relativity is determined by that of a free axially-symmetric scalar field along arbitrary axially-symmetric foliations of a fixed flat $2+1$ dimensional spacetime. We investigate if such a dynamics can be defined unitarily within the standard Fock space quantization of the scalar field.

Evolution between two arbitrary slices of an arbitrary foliation of the flat spacetime can be built out of a restricted class of evolutions (and their inverses). The restricted evolution is from an initial flat slice to an arbitrary (in general, curved) slice of the flat spacetime and can be decomposed into (i) 'time' evolution in which the spatial Minkowskian coordinates serve as spatial coordinates on the initial and the final slice, followed by (ii) the action of a spatial diffeomorphism of the final slice on the data obtained from (i). We show that although the functional evolution of (i) is unitarily implemented in the quantum theory, generic spatial diffeomorphisms of (ii) are not. Our results imply that a Tomanaga-Schwinger type functional evolution of quantum cylindrical waves is not a viable concept even though, remarkably, the more limited notion of functional evolution in Kuchař's 'half parametrized formalism' is well-defined.
\end{abstract}

PACS numbers: 04.60.Ds, 04.60.Kz

*Electronic address: cho@rri.res.in

${ }^{\dagger}$ Electronic address: madhavan@rri.res.in 


\section{INTRODUCTION}

Given the difficulties of constructing a quantum theory of gravity, it is of use to build intuition from the study of simpler toy models. While cosmological mini-superspaces [1, 2] have yielded valuable insights, their finite-dimensional nature precludes the occurrence of field-theoretic aspects of quantum gravity. In this regard, vacuum cylindrically symmetric gravitational fields with 2 hypersurface-orthogonal, commuting Killing vectors (one generating translations along ' $Z$ ' and the other, rotations along ' $\Phi$ ') constitute a useful infinitedimensional midi-superspace. Quantization of this cylindrical wave midi-superspace was initiated by Kuchař [3] and further studied by a number of authors [4, 5, 6, 7, 8]. While the latter works deal with quantization after fixing a gauge, our focus here is on aspects of Dirac quantization of cylindrical waves.

Every cylindrical wave solution is determined by a corresponding axially-symmetric solution to the free scalar wave equation on a fixed 3 dimensional flat spacetime. Specifically, the cylindrical wave line element is

$$
d s^{2}=e^{\gamma-\psi}\left(-(d T)^{2}+(d R)^{2}\right)+R^{2} e^{-\psi}(d \Phi)^{2}+e^{\psi}(d Z)^{2}
$$

and that of the flat spacetime is

$$
d s^{2}=-(d T)^{2}+(d R)^{2}+R^{2}(d \Phi)^{2} .
$$

$\psi(R, T)$ is the free scalar field and $\gamma(R, T)$ is its energy in a box of size $R$ at time $T$. Kuchař defined a canonical transformation from the Arnowitt-Deser-Misner (ADM) phase space of cylindrical waves to that of an axially-symmetric parametrized field theory on $2+1$ dimensional flat spacetime and studied the formal Dirac quantization of the latter system [3].

Parametrized field theory (PFT) is free field theory on flat spacetime in a diffeomorphism invariant disguise 9]. It describes field evolution on arbitrary (and in general, curved) foliations of the flat spacetime instead of only the usual flat foliations, by treating the 'embedding variables' which describe the foliation as dynamical variables to be varied in the action in addition to the scalar field. In the present context the coordinates $X^{\alpha}:=(T, R)$ are parametrized by a new set of arbitrary coordinates $x^{\alpha}=(t, r)$ such that for fixed $t, X^{\alpha}(t, r)$ define an axisymmetric spacelike slice in Minkowski spacetime with radial coordinate $r$. General covariance of PFT ensues from the arbitrary choice of $x^{\alpha}$. In the Hamiltonian description, the embedding variables ' $X^{\alpha}$ ' are canonical coordinates and general covariance implies that Hamiltonian evolution from one slice of an axisymmetric foliation to another is generated by constraints.

Indeed, under Kuchař's canonical transformation ${ }^{1}$, appropriate combinations of the Hamiltonian and diffeomorphism constraints of the midi-superspace take the form of the constraints appropriate to PFT, namely,

$$
C_{\alpha}(x):=P_{\alpha}(x)+h_{\alpha}\left[\psi, \pi, X^{\alpha}\right](x) \approx 0,
$$

where $P_{\alpha}$ and $\pi$ are the momenta conjugate to $X^{\alpha}$ and $\psi$, and $h_{\alpha}$ is related to the stressenergy of the scalar field ${ }^{2}$. The gauge fixing conditions $X^{\alpha}=x^{\alpha}{ }^{3}$ map the classical

\footnotetext{
${ }^{1}$ While $R$ is directly related to the radial components of the metric 1.11, Kuchař's canonical transformation identifies $T$ with the spatial integral of one of the extrinsic curvature components of the cylindrical wave metric (1.1) on a $t=$ constant slice.

${ }^{2}$ As is standard in constraint dynamics, $\approx$ denotes weak equality.
} 
theory directly onto the standard description of a free scalar field in Minkowskian coordinates $(R, T)$. This description admits the standard Heisenberg picture based Fock quantization [3] [4].

In the Dirac quantization, a formal operator version of $C_{\alpha}$ acting on a physical state $|\Psi\rangle$ of the theory is given by

$$
\left(\frac{1}{i} \frac{\delta}{\delta X^{\alpha}}+\hat{h}_{\alpha}\right)|\Psi\rangle=0 .
$$

Eq.(1.4) takes the form of a functional Schrödinger equation which represents infinitesimal evolution of the Schrödinger state $|\Psi\rangle$ from one Cauchy slice to another. In this work we investigate if the formal equation (1.4) is well defined in the context of the standard Heisenberg picture based Fock space quantization of the free scalar field on flat spacetime. More precisely, in analogy with the relation between the Heisenberg and Schrödinger pictures in quantum mechanics, we investigate if there exists a unitary transformation on the standard Fock space which implements evolution between any two axisymmetric Cauchy slices of the flat spacetime and whose infinitesimal version yields equation (1.4).

What do we mean by evolution between two slices? Since the classical theory is that of a free scalar field we explicitly (and uniquely) know the evolved data $(\psi, \pi)$ on the slice $t=t_{1}$ in terms of initial data at $t=t_{0}$. Since the equations for scalar field evolution are linear, we also know how the corresponding quantum operators are related. This is what we mean by evolution. Since this notion of evolution is independent of the choice of interpolating foliation between the two slices, our subsequent considerations are phrased solely in terms of data on the two slices.

We enquire if the operators at the initial and final slices are unitarily related. If they are, then in analogy to the definition of the Schrödinger picture from the Heisenberg picture in usual quantum mechanics, we can define the Schrödinger state at any time $t$ as the unitary image of the state at time $t_{0}{ }^{45}$. Techniques appropriate to our investigation here have been developed in [10, 11] for the case of PFT on $(\mathrm{n}+1)$-dimensional flat spacetime in the absence of axisymmetry. That work arose as an effort to rigorously implement the considerations of Kuchař in [9, 12]. The strategy pursued in [10, 11] is as follows. Classical scalar field evolution from an initial flat slice to any final slice is a linear canonical transformation on the scalar field phase space. Instead of phrasing this canonical transformation in terms of the scalar field and its momentum, one can instead use appropriate linear functionals of these fields. The linear functionals used are such that when they are evaluated on the initial slice, they reduce to the familiar mode coefficients (i.e. the classical correspondents of the standard annihilation and creation operators). It then follows (from the fact that evolution is a canonical transformation) that these functionals evaluated on the final slice are related by a Bogolubov transformation to their initial values. The criterion for unitary implementability of a Bogolubov transformation is that the ' $\beta$ ' matrix be Hilbert-Schmidt (i.e. the squares of the absolute values of its diagonal elements should be summable) [13, 14].

\footnotetext{
${ }^{3}$ Technically, this is not a gauge fixing but a 1-parameter family of gauge fixings (one for each value of $t$ ) known as 'deparameterization'.

${ }^{4}$ For a beautiful exposition of the Schrödinger and Heisenberg pictures in a canonical treatment of PFT on $(\mathrm{n}+1)$-dimensional flat spacetime (in the absence of axisymmetry), we refer the reader to 9,12 .

${ }^{5}$ Note that if we restrict attention to only flat slices, the standard Hamiltonian is the generator of the desired unitary transformation. The non-triviality enters solely due to the possibility of evolution along directions which are not isometries of the spacetime.
} 
The results of [10, 11] are as follows. In 1+1 dimensions operator evolution is unitary, the functional Schrödinger picture exists as the unitary image of the standard Heisenbergpicture-based Fock quantization and the infinitesimal version of the unitary transformation on Heisenberg states implies that the Schrödinger states satisfy an equation of the form (1.4) (albeit, with an extra 'anomaly potential' term 10, 12]). In $n+1$ dimensions $(n>1)$, the $\beta$ coefficients for evolution along generic curved-foliations are not Hilbert-Schmidt. Hence the functional Schrödinger picture does not exist (at least in the form usually envisioned, as the unitary image of the Heisenberg picture) and the functional Schrödinger equation cannot be given any obvious meaning. The case of interest here, namely $2+1$ dimensions with fewer degrees of freedom (only the axisymmetric ones), is a tantalizing intermediate case and as we shall demonstrate, we get a suitably 'in between' answer.

Here, our interest is in evolution between two axisymmetric, but otherwise arbitrary, Cauchy slices $\Sigma_{i}$ and $\Sigma_{f}$ in the flat spacetime (1.2). Note that the restriction of the spacetime coordinate $R$ to any axisymmetric spatial slice defines a natural radial coordinate on the slice. We refer to this natural coordinate by the same symbol $R$. In order to accommodate arbitrary reparameterizations $(R, T) \rightarrow(r, t)$, it is necessary to admit arbitrary radial coordinates $r_{f}(R)$ on $\Sigma_{f}$ and $r_{i}(R)$ on $\Sigma_{i}{ }^{6}$. Despite this, it suffices for our purposes, to consider evolution from a fixed initial slice $\Sigma_{0}$, chosen to be the $T=0$ slice coordinatized by the natural radial coordinate $R$, to an arbitrary final slice $\Sigma$, coordinatized by an arbitrary radial coordinate $r=r(R)$. This entails no loss of generality since, if in the quantum theory all evolution is unitary, quantum evolution from any $\Sigma_{i}$ to any $\Sigma_{f}$ may be constructed as the (inverse) unitary evolution from $\Sigma_{i}$ to $\Sigma_{0}$ followed by unitary evolution from $\Sigma_{0}$ to $\Sigma_{f}$.

Now, consider the restricted evolution from $\Sigma_{0}$ to $\Sigma$ such that the radial coordinate $r$ on $\Sigma$ is chosen to be the natural radial coordinate $R$ i.e. $r$ is identified with the restriction of the spacetime coordinate $R$ to $\Sigma$. We shall refer to such evolution as the 'half parametrized' evolution [3]. Next, we reconsider the general case of interest, namely that of evolution from $\Sigma_{0}$ (with coordinate $R$ ) to $\Sigma$ with coordinate $r=r(R)$. The uniqueness of evolution from initial data implies that $(\psi(r), \pi(r))$ on $\Sigma$ can be obtained by first evolving the initial data from $\Sigma_{0}$ to $\Sigma$ by a 'half parametrized' evolution and then subjecting the result to the spatial diffeomorphism defined by $r(R)$.

In view of this we proceed as follows. In section II we construct the Bogolubov transformation corresponding to the most general evolution from $\Sigma_{0}$ to $\Sigma$. In section III, we particularize the Bogolubov transformation of section II to the case of 'half parametrized' evolution defined above and show that the $\beta$ matrix is Hilbert-Schmidt. This implies that the finite functional evolution corresponding to Kuchař's 'half parameterized' formalism [3] is well defined. In section IV we show that the action of a generic spatial diffeomorphism on data on any slice $\Sigma$ is not unitarily implemented in quantum theory. This implies that the most general Tomonaga- Schwinger type functional evolution of quantum cylindrical waves is not a viable concept. In section $\mathrm{V}$ we digress from the main theme of this paper and turn our attention to the generators of spatial diffeomorphisms of $\Sigma_{0}$. Specifically, we show that such generators generically do not have a well-defined action on the Fock vacuum. Section VI contains a discussion of our results and of open issues. Some technicalities are collected in the Appendices.

\footnotetext{
${ }^{6}$ Here $\Sigma_{i}$ and $\Sigma_{f}$ are slices of constant $t$, i.e. $t=t_{i}$ and $t=t_{f}$. Hence $r$ is the radial coordinate on $\Sigma_{i}$ and $\Sigma_{f}$. Since $r$ is in general different from the coordinate $R, r=r_{i}(R)$ and $r=r_{f}(R)$ express the functional relation between the two coordinates on $\Sigma_{i}$ and on $\Sigma_{f}$.
} 
In what follows we set $\hbar=c=1$. Note that in our discussion of the relation between cylindrical waves and PFT, Newton's constant "per unit Z" [4] has been set to $\frac{1}{8}$.

\section{Note on notations}

The quantum counterpart of a classical quantity, $X$, is denoted by $\hat{X}$, its complex conjugate is denoted by $X^{*}$. $\partial_{t} X, \partial_{r} X$ are denoted by $\dot{X}$ and $X^{\prime}$, respectively. The adjoint of $\hat{X}$ is $\hat{X}^{\dagger}$ and its normal-ordered version is : $\hat{X}: . f(y, k)=O\left(1 / k^{n}\right)$ means that, for $k \rightarrow \infty$, $\exists$ a positive number $C$ such that $|f(y, k)| \leq C / k^{n}$, with $C$ independent of $y$.

\section{EVOLUTION AS A BOGOLUBOV TRANSFORMATION}

The axisymmetric free scalar field, $\psi(R, T)$, satisfies the wave equation on the flat spacetime of equation (1.2). Hence, we have that

$$
-\frac{\partial^{2} \psi}{\partial T^{2}}+\frac{\partial^{2} \psi}{\partial R^{2}}+\frac{1}{R} \frac{\partial \psi}{\partial R}=0
$$

The mode expansion of $\phi$ which is regular at the axis is

$$
\psi(R, T)=\frac{1}{\sqrt{2}} \int_{0}^{\infty} d k J_{0}(k R)\left[a(k) e^{-i k T}+a^{*}(k) e^{i k T}\right] .
$$

Consider an arbitrary axisymmetric foliation of the flat spacetime (1.2) coordinatized by $(t, r)$ (each leaf of the foliation is at constant $t ; r$ is the radial coordinate on each leaf). Let $\Sigma$ be some slice at time $t$. The momentum, $\pi(r, t)$, (canonically conjugate to the scalar field $\psi(r, t)$ on this slice) obtained through a Hamiltonian decomposition of the free scalar field action with respect to the foliation [3] is

$$
\pi=\frac{R}{\left(\dot{T} R^{\prime}-\dot{R} T^{\prime}\right)}\left[\left(R^{\prime 2}-T^{2}\right) \dot{\psi}-\left(\dot{R} R^{\prime}-\dot{T} T^{\prime}\right) \psi^{\prime}\right] .
$$

Here $(R=R(t, r), T=T(t, r))$ denote the spacetime coordinates $(R, T)$ evaluated at the point $(r, t)$ on the foliation. As is conventional in the Hamiltonian description, we shall suppress the label $t$ and denote the scalar field and its momentum on $\Sigma$ by $(\psi(r), \pi(r))$ and the restriction of the spacetime coordinates $R, T$ to $\Sigma$ by $R(r), T(r)$. A useful form of $\pi(r)$ for our subsequent considerations is obtained by re-expressing the $r, t$ derivatives of $\psi$ in Eq. (2.3) in terms of $R, T$ derivatives so that

$$
\pi(r)=R\left(R^{\prime} \psi,_{T}+T^{\prime} \psi, R\right),
$$

where $\psi,_{T}$ and $\psi,_{R}$ denote derivatives of $\psi$ with respect to $T$ and $R$ respectively.

Next, we trade $(\psi(r), \pi(r))$ for suitable linear functionals thereof. We require that these functionals reduce to the mode coefficients $a(k)$ of Eq. (2.2) when $\Sigma$ is chosen to be the flat slice $\Sigma_{0}$ at $T=0$ with radial coordinate $r=R$. Note that from Eq.(2.2) and Eq.(2.4), the field and the momenta on $\Sigma_{0}, \psi_{0}$ and $\pi_{0}$, are

$$
\begin{aligned}
& \psi_{0}=\frac{1}{\sqrt{2}} \int_{0}^{\infty} d k J_{0}(k r)\left[a(k)+a^{*}(k)\right], \\
& \pi_{0}=-\frac{i}{\sqrt{2}} r \int_{0}^{\infty} d k k J_{0}(k r)\left[a(k)-a^{*}(k)\right] .
\end{aligned}
$$


Using the orthogonality of Bessel functions we can invert Eq.(2.5) to get

$$
a(k)=\frac{1}{\sqrt{2}} \int_{0}^{\infty} d r\left[i J_{0}(k r) \pi_{0}(r)-r J_{1}(k r) \psi_{0}^{\prime}(r)\right] .
$$

The evolution from $\Sigma_{0}$ to $\Sigma$ of $a(k)=a(k)\left[\psi_{0}, \pi_{0}\right]$ (where the right hand side of the equality represents the functional of $\psi_{0}, \pi_{0}$ appearing on the right hand side of Eq.(2.6) ) is obtained by replacing the fields $\psi_{0}, \pi_{0}$ by their evolved images $\psi(r), \pi(r)$ on $\Sigma$. Thus we have that (in obvious notation)

$$
a_{\Sigma}(k)=\frac{1}{\sqrt{2}} \int_{0}^{\infty} d r\left[i J_{0}(k r) \pi(r)-r J_{1}(k r) \psi^{\prime}(r)\right] .
$$

Using Eq.(2.2) and Eq.(2.4), $\psi(r), \pi(r)$ can be re-expressed in terms of $a(k), a^{*}(k)$ to obtain an equation of the form

$$
a_{\Sigma}\left(k_{1}\right)=\int_{0}^{\infty} d k_{2}\left[\alpha\left(k_{1}, k_{2}\right) a\left(k_{2}\right)+\beta\left(k_{1}, k_{2}\right) a^{*}\left(k_{2}\right)\right] .
$$

Although we do not display the calculations here, it can be checked that $\alpha$ and $\beta$ satisfy the standard Bogolubov conditions (see for example [15]). On general grounds this is expected since, as mentioned earlier, evolution is a canonical transformation. $\beta\left(k_{1}, k_{2}\right)$ is given by

$$
\begin{aligned}
\beta\left(k_{1}, k_{2}\right) & =\frac{k_{2}}{2} \int_{0}^{\infty} d r r\left(\left[J_{1}\left(k_{1} r\right) J_{1}\left(k_{2} R\right)-\left(\frac{R}{r}\right) J_{0}\left(k_{1} r\right) J_{0}\left(k_{2} R\right)\right] R^{\prime}\right. \\
& \left.-i\left[\left(\frac{R}{r}\right) J_{0}\left(k_{1} r\right) J_{1}\left(k_{2} R\right)+J_{1}\left(k_{1} r\right) J_{0}\left(k_{2} R\right)\right] T^{\prime}\right) e^{i k_{2} T} .
\end{aligned}
$$

Since we do not need the expression for $\alpha$ in this work, we do not display it here.

From the work of Shale [13], the canonical transformation defined by Eq. (2.8) is unitarily implemented iff $\beta$ satisfies the Hilbert- Schmidt condition

$$
\int_{0}^{\infty} d k_{1} \int_{0}^{\infty} d k_{2}|\beta|^{2}<\infty
$$

We end this section with a specification of the class of embeddings $(T(r), R(r))$ for which we analyse (2.10). We require that the embeddings be 'Minkowskian' near the axis of symmetry, $r=0$, and at spatial infinity. Specifically, we require that $R(r)-r$ and $T^{\prime}(r)$ be compactly supported away from $r=0$ and $r \rightarrow \infty$. Hence, on every slice $\Sigma$ we require that there exist positive $a, b, c, d$ with $b>a, d>c$ such that

$$
\begin{gathered}
T^{\prime}(r)=0 \text { for } r \in[0, a) \cup(b, \infty), \\
R(r)-r=0 \text { for } r \in[0, c) \cup(d, \infty) .
\end{gathered}
$$

Note that (2.11) and (2.12) ensure that the slice $\Sigma$ does not have a 'cusp' at the axis.

For future reference, we note that the spatial nature of $\Sigma$ implies that

$$
R^{\prime 2}-T^{2}>0
$$

In particular, the above equation in conjunction with (2.12) implies that

$$
R^{\prime}>0
$$

so that $R$ can indeed be used as a radial coordinate on $\Sigma$. Finally, we shall also assume that $R, T$ are $C^{\infty}$-functions of $r$. 


\section{THE 'HALF PARAMETRIZED' EVOLUTION}

In this section we show that the 'half parametrized' evolution satisfies the Hilbert-Schmidt condition, Eq.(2.10). As mentioned in the introduction, 'half parametrized' evolution is defined as evolution in which the radial coordinate ' $r$ ' on $\Sigma$ is chosen to be the restriction of the spacetime coordinate ' $R$ ' to $\Sigma$. Setting $R=r$ in Eq.(2.9), we get

$$
\begin{aligned}
\beta\left(k_{1}, k_{2}\right) & =\frac{k_{2}}{2} \int_{0}^{\infty} d r r\left(\left[J_{1}\left(k_{1} r\right) J_{1}\left(k_{2} r\right)-J_{0}\left(k_{1} r\right) J_{0}\left(k_{2} r\right)\right]\right. \\
& \left.-i\left[J_{0}\left(k_{1} r\right) J_{1}\left(k_{2} r\right)+J_{1}\left(k_{1} r\right) J_{0}\left(k_{2} r\right)\right] T^{\prime}\right) e^{i k_{2} T} .
\end{aligned}
$$

For later calculations, however, a more symmetric form of $\beta$ turns out to be convenient. As shown in Appendix [C. using Bessel function identities, $\frac{d}{d z}\left[z^{n} J_{n}(z)\right]=z^{n} J_{n-1}(z), \frac{d}{d z} J_{0}(z)=$ $-J_{1}(z)$, their orthogonality relations, and integration by parts in conjunction with Eq.(2.11), we get

$$
\beta\left(k_{1}, k_{2}\right)=-\frac{i}{2} \frac{k_{1} k_{2}}{\left(k_{1}+k_{2}\right)} \int_{a}^{b} d r r\left[J_{0}\left(k_{1} r\right) J_{1}\left(k_{2} r\right)+J_{1}\left(k_{1} r\right) J_{0}\left(k_{2} r\right)\right] T^{\prime} e^{i k_{2} T} .
$$

Clearly, divergent contributions to Eq.(2.10) may be obtained only when $k_{1}$ or $k_{2}$ or both diverge. We estimate these 'ultra-violet' contributions to the integral in (2.10) by considering the following four exhaustive cases.
A.1. $k_{2} \rightarrow \infty, 0<k_{1}<k_{2}^{1 / 3}$.
A.2. $k_{1} \rightarrow \infty, 0<k_{2}<k_{1}^{1 / 3}$.
B.1. $k_{2}, k_{1} \rightarrow \infty, \quad k_{2}^{1 / 3} \leq k_{1} \leq k_{2}$.
B.2. $k_{1}, k_{2} \rightarrow \infty, k_{1}^{1 / 3} \leq k_{2} \leq k_{1}$.

[Case A.1] $k_{2} \rightarrow \infty ; 0<k_{1}<k_{2}^{1 / 3} ; \quad k_{2} a \gg 1$.

Since $k_{2} a \gg 1$, we use the asymptotic expansion of Bessel functions, Appendix $\mathrm{B}$, for the ones with $k_{2} r$ as their argument. The integral contributing to the first term in Eq.(3.2) is

$$
\begin{array}{rl}
\int_{a}^{b} & d r \quad r J_{0}\left(k_{1} r\right) J_{1}\left(k_{2} r\right) T^{\prime} e^{i k_{2} T} \\
& =\frac{1}{\sqrt{2 \pi k_{2}}} \int_{a}^{b} d r \sqrt{r} T^{\prime} J_{0}\left(k_{1} r\right)\left\{e^{-i 3 \pi / 4} e^{i k_{2}(r+T)}+e^{i 3 \pi / 4} e^{-i k_{2}(r-T)}+O\left(\frac{1}{k_{2}}\right)\right\} .
\end{array}
$$

Since $T^{\prime}$ has compact support away from the origin, $\sqrt{r} T^{\prime} J_{0}\left(k_{1} r\right)$ is a smooth, bounded function of compact support. Further, for space-like embeddings, $(r \pm T)^{\prime} \neq 0$ because $\left|T^{\prime}\right|<1$ (this follows from Eq.(2.13) with $R=r$ ). Then the integral in Eq.(3.3) is a linear combination of integrals of the form considered in Lemma 1 with $f_{3}=0$. A similar analysis yields the same conclusion for the second term in Eq.(3.2). Hence, using Lemma 1, $\beta$ can be estimated as

$$
\left|\beta\left(k_{1}, k_{2}\right)\right|<\frac{k_{1} k_{2}}{\left(k_{1}+k_{2}\right)}\left[\left(\frac{k_{1}}{k_{2}^{3 / 2}} M_{1}+\frac{1}{k_{2}^{3 / 2}} M_{2}\right)\right],
$$


for suitable positive real numbers $M_{1}, M_{2}$. It follows that

$$
\left|\beta\left(k_{1}, k_{2}\right)\right|^{2}<\frac{k_{1}^{2}}{k_{2}^{3}}\left[C_{1}+C_{2} k_{1}+C_{3} k_{1}^{2}\right],
$$

for suitable $C_{1}, C_{2}, C_{3}$. This gives the estimate

$$
\int_{0}^{k_{2}^{1 / 3}} d k_{1}\left|\beta\left(k_{1}, k_{2}\right)\right|^{2}<\frac{C_{1}}{3} \frac{1}{k_{2}^{2}}+\frac{C_{2}}{4} \frac{1}{k_{2}^{5 / 3}}+\frac{C_{3}}{5} \frac{1}{k_{2}^{4 / 3}},
$$

which in turn implies convergence of the integral in Eq.(2.10) in the domain of integration considered here.

[Case A.2] $k_{1} \rightarrow \infty ; 0<k_{2}<k_{1}^{1 / 3} ; \quad k_{1} a \gg 1$.

The only non-symmetric part (with respect to $k_{1}$ and $k_{2}$ ) of $\beta\left(k_{1}, k_{2}\right)$ in Eq.(3.2) is $e^{i k_{2} T}$, which results in the terms $e^{i\left(k_{2} T \pm k_{1} r\right)}$ rather than $e^{i k_{2}(r \pm T)}$ in Eq.(13.3). However, Lemma 1 still applies. The rest of the argument is similar to the one for [Case A.1], and also yields a convergent contribution to Eq.(2.10) in this domain of integration.

[Case B.1] $k_{2}, k_{1} \rightarrow \infty ; k_{2}^{1 / 3} \leq k_{1} \leq k_{2} ; \quad k_{1} a, k_{2} a \gg 1$.

In this case we can expand all the Bessel functions using their asymptotic forms for large arguments. Then we have that,

$$
\begin{aligned}
\beta\left(k_{1}, k_{2}\right) & =\frac{i}{8 \pi} \frac{\left(k_{1} k_{2}\right)^{1 / 2}}{\left(k_{1}+k_{2}\right)} \int_{a}^{b} d r\left[8 \cos \left(k_{1}+k_{2}\right) r-\left[\sin \left(k_{1}+k_{2}\right) r\right.\right. \\
& \left.\left.+2 \cos \left(k_{1}-k_{2}\right) r\right]\left(\frac{1}{k_{1}}+\frac{1}{k_{2}}\right) \frac{1}{r}+O\left(\frac{1}{k_{1}^{2}}\right)\right] T^{\prime} e^{i k_{2} T} \\
& :=\beta_{+}+\beta_{-}+K .
\end{aligned}
$$

Here $\beta_{+}$is the sum of the first two terms (i.e. the $\cos \left(k_{1}+k_{2}\right) r$ and the $\sin \left(k_{1}+k_{2}\right) r$ terms), while $\beta_{-}$is the third term (with the $\cos \left(k_{1}-k_{2}\right) r$ ). $K$ denotes the remainder of order $1 / k_{1}^{2}$.

First we show that $\beta_{+}$falls off faster than any power of $\frac{1}{\left(k_{1}+k_{2}\right)}$. The two terms in $\beta_{+}$are of the form

$$
f_{ \pm}\left(k_{1}, k_{2}\right) \int_{a}^{b} d r g_{ \pm}(r)\left[e^{i\left(k_{1}+k_{2}\right) r} \pm e^{-i\left(k_{1}+k_{2}\right) r}\right] T^{\prime} e^{i k_{2} T}
$$

where $g_{ \pm}(r), T(r)$ are smooth bounded functions of $r$ in $[a, b]$. Consider the first term in (3.9). Define $\chi(r):=T(r)+r, t:=\frac{k_{1}}{k_{1}+k_{2}}$ and $\chi_{t}$ as

$$
\chi_{t}:=t r+(1-t) \chi(r) .
$$

Since $\left|T^{\prime}\right|<1$ for a space-like embedding, $\chi_{t}^{\prime} \neq 0$ in the domain of the integration. This implies that there exists a $C^{\infty}$ function $\psi_{t}(y)=r$ where $\chi_{t}(r)=y$. Then,

$$
\int_{a}^{b} d r g_{ \pm}(r) e^{i\left(k_{1}+k_{2}\right) r} T^{\prime} e^{i k_{2} T}=\int_{\chi_{t}(a)}^{\chi_{t}(b)} d y g_{ \pm}(r(y)) \frac{d \psi_{t}}{d y} T^{\prime}(r(y)) e^{i y\left(k_{1}+k_{2}\right)}
$$


This is an integral of the form in Lemma 2, L.2.a. Therefore the integral falls off faster than any power of $\frac{1}{\left(k_{1}+k_{2}\right)}$ in the large $k_{1}$ and $k_{2}$ limit. Similar arguments apply to the second term in (3.9), so we conclude that $\beta_{+}$falls off faster than any power of $\frac{1}{\left(k_{1}+k_{2}\right)}$ in the large $k_{1}, k_{2}$ limit.

Next, consider $K$. Since $k_{2}+k_{1}>k_{2}$ and $T^{\prime}$ is bounded

$$
|K|=\left|\frac{i}{8 \pi} \frac{\left(k_{1} k_{2}\right)^{1 / 2}}{\left(k_{1}+k_{2}\right)} \int_{a}^{b} d r T^{\prime} e^{i k_{2} T} O\left(\frac{1}{k_{1}^{2}}\right)\right|<\frac{C_{4}}{k_{2}^{1 / 2} k_{1}^{3 / 2}}
$$

with a suitable positive $C_{4}$.

Finally, consider $\beta_{-}$,

$$
\beta_{-}=-\frac{i}{4 \pi\left(k_{1} k_{2}\right)^{1 / 2}} \int_{a}^{b} d r \cos \left(\left(k_{1}-k_{2}\right) r\right) \frac{T^{\prime}}{r} e^{i k_{2} T} .
$$

Since the integrand is bounded, a rough estimate for $\beta_{-}$yields $\left|\beta_{-}\right|=O\left(\left(k_{1} k_{2}\right)^{-1 / 2}\right)$. From Eq.(3.8)

$$
|\beta|^{2} \leq\left|\beta_{+}\right|^{2}+\left|\beta_{-}\right|^{2}+|K|^{2}+2\left|\beta_{+} \beta_{-}\right|+2\left|\beta_{+} K\right|+2\left|\beta_{-} K\right| .
$$

Since $\left|\beta_{+}\right|$falls off faster than any power of $\frac{1}{\left(k_{1}+k_{2}\right)}$, so do $\left|\beta_{+}\right|^{2},\left|\beta_{+} \beta_{-}\right|$, and $\left|\beta_{+} K\right|$. Hence these terms give convergent contributions to the integral in Eq.(2.10).

Next, from Eq.(3.12), and $\beta_{-}=O\left(\left(k_{1} k_{2}\right)^{-1 / 2}\right)$, we estimate the contributions from $|K|^{2}$ and $2\left|\beta_{-} K\right|$ as

$$
\int_{k_{2}^{1 / 3}}^{k_{2}} d k_{1}|K|^{2}<\frac{C_{4}^{2}}{k_{2}^{5 / 3}}
$$

and

$$
\int_{k_{2}^{1 / 3}}^{k_{2}} d k_{1}\left|\beta_{-} K\right|<\frac{C_{5}}{k_{2}^{4 / 3}},
$$

for some appropriate positive constant $C_{5}$, which lead to convergent contributions to the integral in Eq.(2.10).

So far all the contributions to Eq.(2.10) except the one from $\left|\beta_{-}\right|^{2}$ have been shown to be convergent. The rough estimate of $\beta_{-}=O\left(\left(k_{1} k_{2}\right)^{-1 / 2}\right)$, used above, however, seems to indicate that this contribution diverges. But, as we shall show now, a better estimate of $\beta_{-}$ yields a convergent contribution.

We introduce an auxiliary variable $\alpha:=\frac{k_{2}-k_{1}}{k_{2}}$. Clearly, $0 \leq \alpha<1$. $\beta_{-}$is then given by

$$
\beta_{-}=-\frac{i}{8 \pi\left(k_{1} k_{2}\right)^{1 / 2}} \int_{a}^{b} d r \frac{T^{\prime}}{r}\left[e^{i k_{2}(T-\alpha r)}+e^{i k_{2}(T+\alpha r)}\right] .
$$

Since $\left|T^{\prime}\right|<1$ and $[a, b]$ is compact, there exists a number $\bar{m}$ such that $\left|T^{\prime}\right| \leq \bar{m}<1$. Furthermore, let $m:=\bar{m}+\frac{1-\bar{m}}{2}, m>\bar{m}$. Consider Eq.(3.17) when $\alpha>m$. If we naively estimate the integral in Eq.(3.17) as of $O(1)$, the prefactor, $\left(k_{1} k_{2}\right)^{-1 / 2}=O\left(1 / k_{2}^{2 / 3}\right)$, gives divergent contribution to Eq.(2.10). However, since $T^{\prime} \pm \alpha \neq 0$, the integral falls off faster than any power of $1 / k_{2}$ by Lemma 2 L.2.a. Thus, both terms in Eq.(3.17) give convergent contributions. 
The remaining contribution to be estimated is when $\alpha \leq m$ (That is, $\left.(1-m) k_{2} \leq k_{1}\right)$. We obtain, for an appropriate constant $C, C>0$, for $0 \leq \alpha \leq m$,

$$
\left|\beta_{-}\right|^{2} \leq \frac{C}{k_{2}^{2}} \int_{a}^{b} \int_{a}^{b} d r_{1} d r_{2} \frac{T^{\prime}\left(r_{1}\right) T^{\prime}\left(r_{2}\right)}{r_{1} r_{2}} e^{i k_{2}\left[T\left(r_{1}\right)-T\left(r_{2}\right)\right]}\left[\cos \alpha k_{2}\left(r_{1}+r_{2}\right)+\cos \alpha k_{2}\left(r_{1}-r_{2}\right)\right] .
$$

Since the prefactor is $O\left(k_{2}^{-2}\right)$, we still need the tiniest power of $1 / k_{2}$ from the integral involving $\cos \alpha k_{2}\left(r_{1} \pm r_{2}\right)$ for convergence. The key point is to change variables from $\left(k_{1}, k_{2}\right)$ to $\left(\alpha, k_{2}\right)$ and integrate $\left|\beta_{-}\right|^{2}$ over $\alpha$. The integral of $\cos k_{2} \alpha\left(r_{1}+r_{2}\right)$ over $\alpha$ gives a factor of $1 / k_{2}$ since $\left(r_{1}+r_{2}\right) \geq a$. Since $d k_{1} d k_{2}=k_{2} d k_{2} d \alpha$, this term contributes $\int k_{2} d k_{2} O\left(k_{2}^{-3}\right)$ which is convergent.

The only remaining non-trivial contribution is from the term with $\cos \alpha k_{2}\left(r_{1}-r_{2}\right)$. From Eq.(3.18) this contribution can be estimated as

$$
\begin{aligned}
\frac{C}{k_{2}^{2}} \int_{0}^{m} d \alpha \int_{a}^{b} \int_{a}^{b} d r_{1} d r_{2} \frac{T^{\prime}\left(r_{1}\right) T^{\prime}\left(r_{2}\right)}{r_{1} r_{2}} e^{i k_{2}\left[T\left(r_{1}\right)-T\left(r_{2}\right)\right]} \cos \alpha k_{2}\left(r_{1}-r_{2}\right) \\
\leq\left|\frac{C}{k_{2}^{2}} \int_{a}^{b} \int_{a}^{b} d r_{1} d r_{2} \frac{T^{\prime}\left(r_{1}\right) T^{\prime}\left(r_{2}\right)}{r_{1} r_{2}} e^{i k_{2}\left[T\left(r_{1}\right)-T\left(r_{2}\right)\right]} \int_{0}^{m} d \alpha \cos \alpha k_{2}\left(r_{1}-r_{2}\right)\right| \\
\leq \frac{C}{k_{2}^{2}} \int d r_{+} d r_{-} f\left(r_{-}, r_{+}\right)\left|\frac{\sin k_{2} r_{-} m}{k_{2} r_{-}}\right| \\
=\frac{C}{k_{2}^{2}} \int d r_{+} \int_{r_{-}>0} d r_{-} f\left(r_{-}, r_{+}\right)\left|\frac{\sin k_{2} r_{-} m}{k_{2} r_{-}}\right| \\
\quad+\frac{C}{k_{2}^{2}} \int d r_{+} \int_{r_{-}<0} d r_{-} f\left(r_{-}, r_{+}\right)\left|\frac{\sin k_{2} r_{-} m}{k_{2} r_{-}}\right| \\
:=I_{I}+I_{I I} .
\end{aligned}
$$

where $r_{-}:=r_{1}-r_{2}, r_{+}:=r_{1}+r_{2}$ and $f\left(r_{-}, r_{+}\right) ;=\left|\frac{T^{\prime}\left(r_{1}\right) T^{\prime}\left(r_{2}\right)}{2 r_{1} r_{2}}\right|$ is a positive function of compact support in $\left(r_{1}, r_{2}\right)$, and hence in $\left(r_{-}, r_{+}\right)$plane. Now we estimate $I_{I}$,

$$
\begin{aligned}
I_{I} & =\frac{C}{k_{2}^{2}} \int d r_{+}\left[m \int_{0}^{1 / k_{2}^{2}} d r_{-} f\left(r_{-}, r_{+}\right)\left|\frac{\sin k_{2} r_{-} m}{k_{2} r_{-} m}\right|+\int_{1 / k_{2}^{2}}^{k_{2}^{1-\epsilon}} d r_{-} f\left(r_{-}, r_{+}\right)\left|\frac{\sin k_{2} r_{-} m}{k_{2} r_{-}}\right|\right. \\
& \left.+\int_{k_{2}^{1-\epsilon}}^{\infty} d r_{-} f\left(r_{-}, r_{+}\right)\left|\frac{\sin k_{2} r_{-} m}{k_{2} r_{-}}\right|\right] .
\end{aligned}
$$

The last integral doesn't contribute since, for $\epsilon<1$ and large enough $k_{2}, f\left(r_{-}, r_{+}\right)=0$ because $f\left(r_{-}, r_{+}\right)$is of compact support. The term $\left|\frac{\sin k_{2} r_{-} m}{k_{2} r_{-} m}\right|$ in the first integral can also be bounded because it's maximum is 1 . Then,

$$
\begin{aligned}
I_{I} & <\frac{C}{k_{2}^{2}} m \int_{r_{+}<B} d r_{+} \int_{0}^{1 / k_{2}^{2}} d r_{-} f_{\max }+\frac{C}{k_{2}^{3}} \int_{r_{+}<B} d r_{+} \int_{1 / k_{2}^{2}}^{k_{2}^{1-\epsilon}} d r_{-} \frac{f_{\max }}{r_{-}} \\
& \leq \frac{D}{k_{2}^{4}}+\frac{E \ln k_{2}}{k_{2}^{3}}
\end{aligned}
$$

where $f_{\max }=\max f\left(r_{-}, r_{+}\right)$, and $f=0$ for $r_{+} \geq B$. D, E are appropriate constants. By redefining $r_{-}$to $\bar{r}_{-}:=-r_{-}$we get the same expression for $I_{I I}$, and therefore the same 
estimate. This implies that,

$$
\int_{0}^{m} d \alpha\left|\beta_{-}\right|^{2}<M \frac{\ln k_{2}}{k_{2}^{3}}
$$

for an appropriate M, which again leads to a convergent contribution. Since all the terms in Eq.(3.14) are finally shown to give convergent contributions, the integral in Eq.(2.10) is convergent.

[Case B.2] $k_{2}, k_{1} \rightarrow \infty ; k_{1}^{1 / 3} \leq k_{2} \leq k_{1} ; \quad k_{1} a, k_{2} a \gg 1$

Using methods similar to those in [Case B.1] it can be checked that, once again, the only potentially divergent contribution is from $\left|\beta_{-}\right|^{2}$ with $\beta_{-}$defined as before, by Eq.(13.13).

To estimate this contribution, we again introduce an auxiliary variable $\alpha:=\frac{k_{1}-k_{2}}{k_{2}}>0$ (Note that $\alpha$ in the [Case B.2] is different from the $\alpha$ in the [Case B.1] even though we use the same symbol.). First, consider the range $0 \leq \alpha \leq m$ ( $m$ has already been defined, see [Case B.1]). Then, as in the [case B.1] we get, for a suitable constant $\tilde{C}, \tilde{C}>0$,

$$
\left|\beta_{-}\right|^{2} \leq \frac{\tilde{C}}{k_{2}^{2}} \int_{a}^{b} \int_{a}^{b} d r_{1} d r_{2} \frac{T^{\prime}\left(r_{1}\right) T^{\prime}\left(r_{2}\right)}{r_{1} r_{2}} e^{i k_{2}\left[T\left(r_{1}\right)-T\left(r_{2}\right)\right]}\left[\cos \alpha k_{2}\left(r_{1}+r_{2}\right)+\cos \alpha k_{2}\left(r_{1}-r_{2}\right)\right]
$$

which is exactly the same form as in Eq.(3.18). The rest follows exactly as in [Case B.1] and the contribution to (2.10) from $\left|\beta_{-}\right|^{2}$ for $0 \leq \alpha \leq m$ is convergent.

The only remaining case is when $\alpha>m$. We define another auxiliary variable $\bar{\alpha}:=$ $\frac{k_{1}-k_{2}}{k_{1}}=\frac{k_{2}}{k_{1}} \alpha$. In the range of $k_{1}, k_{2}$ considered with $\alpha>m, \frac{m}{m+1}<\bar{\alpha}<1$. This implies that $0<1-\bar{\alpha}<(m+1)^{-1}$. In terms of $\bar{\alpha}$ we have,

$$
\beta_{-}=-\frac{i}{8 \pi\left(k_{1} k_{2}\right)^{1 / 2}} \int_{a}^{b} d r \frac{T^{\prime}}{r}\left[e^{i k_{1}[(1-\bar{\alpha}) T(r)+\bar{\alpha} r]}+e^{i k_{1}[(1-\bar{\alpha}) T(r)-\bar{\alpha} r]}\right],
$$

where $T^{\prime} / r$ is a smooth function of compact support. Let $f_{ \pm}=(1-\bar{\alpha}) T \pm \bar{\alpha} r . f_{ \pm}^{\prime} \neq 0$ because $\left|(1-\bar{\alpha}) T^{\prime}\right|<(1-\bar{\alpha}) m<\frac{m}{m+1}<\bar{\alpha}$. Therefore, both terms in the integral fall off faster than any power of $1 / k_{1}$ using Lemma 2 L.2.a. Hence, this contribution from $\beta_{-}$to (2.10) is convergent. This concludes our analysis of [Case B.2].

Since the integral in Eq.(2.10) with $\beta$ given in Eq.(3.2) converges in all domains of integration, we conclude that $\beta$ for the half-parametrized case is Hilbert-Schmidt.

\section{ACTION OF SPATIAL DIFFEOMORPHISMS}

As discussed in the Introduction, evolution from $\Sigma_{0}$ (with radial coordinate ' $R$ ') to an arbitrary axisymmetric slice $\Sigma$ (with radial coordinate ' $r$ ') is a composition of 'half parametrized' evolution followed by the action of a spatial diffeomorphism on the final slice. Since 'half parametrized' evolution is unitary (see section III), evolution from $\Sigma_{0}$ to $\Sigma$ is unitary iff the action of spatial diffeomorphisms on $\Sigma$ is unitary. As we show in this section, however, the action of a generic spatial diffeomorphism on an arbitrary Cauchy slice is not of HilbertSchmidt type. Therefore, even though 'half parametrized' evolution is of Hilbert-Schmidt type, generic evolution between $\Sigma_{0}$ and $\Sigma$ is not. 
We proceed as follows. First, we show that the expression for $\beta\left(k_{1}, k_{2}\right)$ for a diffeomorphism on $\Sigma$ is identical to that for $\beta\left(k_{1}, k_{2}\right)$ for a corresponding diffeomorphism on $\Sigma_{0}$. Next, we show that the explicit expression for $\beta\left(k_{1}, k_{2}\right)$ for generic diffeomorphisms of $\Sigma_{0}$ is not Hilbert-Schmidt.

Consider a slice $\Sigma$ with coordinate system $R$ (see the remark after Eq.(2.14)). Let ' $d$ ' be a diffeomorphism from $\Sigma$ to itself which maps the point labelled by $R$ to the point labelled by $r(R)$. Explicitly, a point with coordinate $R=R_{0}$ is mapped to a point with coordinate $R=r\left(R_{0}\right)$. The action of ' $d$ ' on Cauchy data $(\psi, \pi)$ on $\Sigma$ is a linear canonical transformation which can be written as

$$
\begin{aligned}
& (\psi)_{d}(r)=\int_{0}^{\infty} d \tilde{r} g_{d}(\tilde{r}, r) \psi(\tilde{r}) \\
& (\pi)_{d}(r)=\int_{0}^{\infty} d \tilde{r} h_{d}(\tilde{r}, r) \pi(\tilde{r}) .
\end{aligned}
$$

Since ' $d$ ' drags the fields at $R$ to $r(R)$, we have that $g_{d}(\tilde{r}, r)=h_{d}(\tilde{r}, r)=\delta(\tilde{r}, R(r))$.

Next, consider the initial slice $\Sigma_{0}$ also with coordinate system $R$. Let ' $d_{0}$ ' be the diffeomorphism from $\Sigma_{0}$ to itself which maps the point labelled by $R$ to the point labelled by $r(R)$. Clearly, the action of ' $d_{0}$ ' on data $\left(\psi_{0}, \pi_{0}\right)$ is

$$
\begin{aligned}
& \left(\psi_{0}\right)_{d_{0}}(r)=\int_{0}^{\infty} d \tilde{r} g_{d_{0}}(\tilde{r}, r) \psi_{0}(\tilde{r}), \\
& \left(\pi_{0}\right)_{d_{0}}(r)=\int_{0}^{\infty} d \tilde{r} h_{d_{0}}(\tilde{r}, r) \pi_{0}(\tilde{r})
\end{aligned}
$$

with $g_{d_{0}}(\tilde{r}, r)=h_{d_{0}}(\tilde{r}, r)=\delta(\tilde{r}, R(r))$. Thus, for every ' $d$ ' on $\Sigma$, there exists ' $d_{0}$ ' on $\Sigma_{0}$ (and vice versa) with the same (slice independent) integral kernels. We shall now show that, for the Bogolubov transformation of interest, $\beta\left(k_{1}, k_{2}\right)$ for ' $d_{0}$ ' is the same as $\beta\left(k_{1}, k_{2}\right)$ for ' $d$ '.

First, consider the calculation of $\beta\left(k_{1}, k_{2}\right)$ corresponding to ' $d_{0}$ '. We compute $\beta\left(k_{1}, k_{2}\right)$ along the lines described in section II. Denote the image of the mode coefficient $a(k)$ under ' $d_{0}$ ' as $a_{d_{0}}(k)$. Then, we have that,

$$
\begin{aligned}
a_{d_{0}}(k) & =\frac{1}{\sqrt{2}} \int_{0}^{\infty} d r\left[i J_{0}(k r)\left(\pi_{0}\right)_{d_{0}}(r)-r J_{1}(k r)\left(\psi_{0}\right)_{d_{0}}^{\prime}(r)\right] \\
& =\frac{1}{\sqrt{2}} \int_{0}^{\infty} d r \int_{0}^{\infty} d \tilde{r}\left[i J_{0}(k r) h_{d_{0}}(\tilde{r}, r) \pi_{0}(\tilde{r})-r J_{1}(k r) g_{d_{0}}^{\prime}(\tilde{r}, r) \psi_{0}(\tilde{r})\right] .
\end{aligned}
$$

Here, we used Eqs.(4.3) and (4.4) in the second line. Finally, we express $\left(\psi_{0}, \pi_{0}\right)$ in the second line in terms of their mode decomposition,

$$
\begin{aligned}
& \psi_{0}(\tilde{r})=\int_{0}^{\infty} d k\left[a(k) u(\tilde{r})+a^{*}(k) u^{*}(\tilde{r})\right] \\
& \pi_{0}(\tilde{r})=\int_{0}^{\infty} d k\left[a(k) v(\tilde{r})+a^{*}(k) v^{*}(\tilde{r})\right],
\end{aligned}
$$

where $u(\tilde{r})=1 / \sqrt{2} J_{0}(k \tilde{r})=u^{*}(\tilde{r})$, and $v(\tilde{r})=-i / \sqrt{2} J_{0}(k \tilde{r})=-v^{*}(\tilde{r})$ (see Eq.(2.5)). This allow us to read $\beta$ from

$$
a_{d_{0}}\left(k_{1}\right)=\int_{0}^{\infty} d k_{2}\left[\alpha\left(k_{1}, k_{2}\right) a\left(k_{2}\right)+\beta\left(k_{1}, k_{2}\right) a^{*}\left(k_{2}\right)\right] .
$$


The computation of $\beta$ for an arbitrary slice $\Sigma$ is an exactly analogous process. The action of ' $d$ ' on data $(\psi, \pi)$ is given by (4.1) and (4.2). Here, $(\psi, \pi)$ is the image of initial data on $\Sigma_{0}$ under half parametrized evolution. In section III we showed that the Bogolubov transformation for half parametrized evolution between $a(k)$ and $a_{\Sigma}(k)$ was Hilbert-Schimidt. It remains to evaluate the action of the canonical transformation (4.1) and (4.2) on these $a_{\Sigma}(k)$. From section II, $a_{\Sigma}(k)$ and $(\psi, \pi)$ are related by

$$
a_{\Sigma}(k)=\frac{1}{\sqrt{2}} \int_{0}^{\infty} d r\left[i J_{0}(k r) \pi(r)-r J_{1}(k r) \psi^{\prime}(r)\right]
$$

and

$$
\begin{aligned}
& \psi(\tilde{r})=\int_{0}^{\infty} d k\left[a_{\Sigma}(k) u(\tilde{r})+a_{\Sigma}^{*}(k) u^{*}(\tilde{r})\right] \\
& \pi(\tilde{r})=\int_{0}^{\infty} d k\left[a_{\Sigma}(k) v(\tilde{r})+a_{\Sigma}^{*}(k) v^{*}(\tilde{r})\right] .
\end{aligned}
$$

From Eqs.(4.1), (4.2) and (4.9), the image of $a_{\Sigma}(k)$ under ' $d$ ' is

$$
\begin{aligned}
\left(a_{\Sigma}\right)_{d}(k) & =\frac{1}{\sqrt{2}} \int_{0}^{\infty} d r\left[i J_{0}(k r)(\pi)_{d}(r)-r J_{1}(k r)(\psi)_{d}^{\prime}(r)\right] \\
& =\frac{1}{\sqrt{2}} \int_{0}^{\infty} d r \int_{0}^{\infty} d \tilde{r}\left[i J_{0}(k r) h_{d}(\tilde{r}, r) \pi(\tilde{r})-r J_{1}(k r) g_{d}^{\prime}(\tilde{r}, r) \psi(\tilde{r})\right] .
\end{aligned}
$$

Substituting Eqs.(4.10) and (4.11) into Eq.(4.12), we can read off $\beta$ from

$$
\left(a_{\Sigma}\right)_{d}\left(k_{1}\right)=\int_{0}^{\infty} d k_{2}\left[\alpha\left(k_{1}, k_{2}\right) a_{\Sigma}\left(k_{2}\right)+\beta\left(k_{1}, k_{2}\right) a_{\Sigma}^{*}\left(k_{2}\right)\right] .
$$

Since $h_{d}=h_{d_{0}}, g_{d}=g_{d_{0}}, \beta$ is identical to the expression obtained when $\Sigma=\Sigma_{0}$. Therefore, without loss of generality, we shall use $\beta$ corresponding to the action of ' $d_{0}$ ' on the initial slice $\Sigma_{0}$.

$\beta\left(k_{1}, k_{2}\right)$ for the initial slice $\Sigma_{0}$ can be easily computed by setting $T=0$, and $T^{\prime}=0$ in Eq.(2.9) so that

$$
\beta\left(k_{1}, k_{2}\right)=\frac{k_{2}}{2} \int_{0}^{\infty} d R\left[r J_{1}\left(k_{1} r\right) J_{1}\left(k_{2} R\right)-R J_{0}\left(k_{1} r\right) J_{0}\left(k_{2} R\right)\right] .
$$

Note that $r J_{1}\left(k_{1} r\right)-R J_{1}\left(k_{1} R\right)$ and $J_{0}\left(k_{1} r\right)-J_{0}\left(k_{1} R\right)$ have support only in $[c, d]$ since $R=r$ outside $[c, d]$, Eq.(2.12). This together with Eq.C3) implies that, for a smooth function $g(R)$ with compact support in $[\bar{c}, \bar{d}]$, where $\bar{c}<c<d<\bar{d}$, such that $g(R)=1$ for $R \in[c, d]$, we can write $\beta$ as

$$
\begin{aligned}
\beta\left(k_{1}, k_{2}\right) & =\frac{k_{2}}{2} \int_{\bar{c}}^{\bar{d}} d R g(R)\left[r J_{1}\left(k_{1} r\right) J_{1}\left(k_{2} R\right)-R J_{0}\left(k_{1} r\right) J_{0}\left(k_{2} R\right)\right] \\
& -\frac{k_{2}}{2} \int_{\bar{c}}^{\bar{d}} d R g(R) R\left[J_{1}\left(k_{1} R\right) J_{1}\left(k_{2} R\right)-J_{0}\left(k_{1} R\right) J_{0}\left(k_{2} R\right)\right] \\
& :=\beta_{[r, R]}\left(k_{1}, k_{2}\right)-\beta_{[R, R]}\left(k_{1}, k_{2}\right) .
\end{aligned}
$$


To show that the integral in Eq.(2.10) with $\beta$ given in (4.15) to (4.17) diverges, it is sufficient to show that the integral diverges over any subset of the domain of integration because the integrand in Eq.(2.10) is always positive. We choose this subset as follows. We change variables from $\left(k_{1}, k_{2}\right)$ to $\left(\alpha:=k_{1} / k_{2}, k_{2}\right)$. Let $\alpha_{0} \in \mathbf{R}$ be such that $\alpha_{0}>0, \alpha_{0} \neq 1$. We shall restrict attention to the subdomain of integration defined by $\alpha_{0}-\delta<\alpha<\alpha_{0}+\delta$ and $k_{2(0)}<k_{2}<2 k_{2(0)}, k_{2(0)} \rightarrow \infty$. Here $\delta$ is chosen such that $\delta \ll \alpha_{0}$ so that, in the range considered, $\alpha>0$ and $\alpha \neq 1$.

Similar to the previous section, we use the asymptotic expansion of Bessel function for large arguments, Appendix B, to estimate $\beta$. We get,

$$
\begin{aligned}
\beta_{[r, R]} & =\frac{1}{4 \pi \alpha^{1 / 2}} \int_{\bar{c}}^{\bar{d}} d R g(R)\left[\frac{r+R}{\sqrt{r R}} i-\frac{(3 r-R)(R+\alpha r)}{8 \alpha \sqrt{(r R)^{3}}} \frac{1}{k_{2}}\right. \\
& +\frac{1}{128}\left(\frac{(15 r-9 R)\left(R^{2}+\alpha^{2} r^{2}\right)}{\alpha^{2} \sqrt{(r R)^{5}}}-\frac{(18 r+2 R)}{\left.\alpha \sqrt{(r R)^{3}}\right)} i \frac{1}{k_{2}^{2}}\right] e^{i k_{2}(R+\alpha r)}+\text { c.c. } \\
& +\frac{1}{4 \pi \alpha^{1 / 2}} \int_{\bar{c}}^{\bar{d}} d R g(R)\left[\frac{r-R}{\sqrt{r R}}-\frac{(3 r+R)(R-\alpha r)}{8 \alpha \sqrt{(r R)^{3}}} i \frac{1}{k_{2}}\right. \\
& +\frac{1}{128}\left(\frac{(15 r+9 R)\left(R^{2}+\alpha^{2} r^{2}\right)}{\alpha^{2} \sqrt{(r R)^{5}}}+\frac{(18 r-2 R)}{\left.\alpha \sqrt{(r R)^{3}}\right)} \frac{1}{k_{2}^{2}}\right] e^{i k_{2}(R-\alpha r)}+\text { c.c. } \\
& +O\left(\frac{1}{k_{2}^{3}}\right) \\
& :=\beta_{[r, R]}^{+}+\beta_{[r, R]}^{-}+O\left(\frac{1}{k_{2}^{3}}\right)
\end{aligned}
$$

where 'c.c' refers to 'complex conjugate', $\beta_{[r, R]}^{+}$is (4.18) and $\beta_{[r, R]}^{-}$is (4.19) $) \beta_{[R, R]}$ in Eq. (4.17) can also be written down in a similar form by replacing $r$ with $R$ in (4.18) and (4.19). Similar to Eq.(4.21) we can also write $\beta_{[R, R]}$ as a sum of $\beta_{[R, R]}^{+}, \beta_{[R, R]}^{-}$and a term of $O\left(1 / k_{2}^{3}\right)$. Then, $\beta$ is,

$$
\beta=\beta_{[r, R]}^{+}+\beta_{[r, R]}^{-}+\beta_{[R, R]}^{+}+\beta_{[R, R]}^{-}+O\left(\frac{1}{k_{2}^{3}}\right) .
$$

Now, we show that $\beta_{[R, R]}^{+}, \beta_{[R, R]}^{-}$, and $\beta_{[r, R]}^{+}$are of rapid decrease in $k_{2}$. Consider $\beta_{[r, R]}^{+}$ first. The integrals in $\beta_{[r, R]}^{+}$are of the form in Lemma 2, L.2.a, because $R^{\prime}>0$ and $\alpha>0$ in the domain of interest implies that

$$
\frac{d}{d R}(R+\alpha r)=1+\alpha \frac{d r}{d R} \neq 0
$$

A similar argument holds for $\beta_{[R, R]}^{+}$. The contribution from $\beta_{[R, R]}^{-}$can also be estimated using Lemma 2, L.2.a. Explicitly, $\beta_{[R, R]}^{-}$is,

$$
\frac{1}{4 \pi \alpha^{1 / 2}} \int_{\bar{c}}^{\bar{d}} d R g(R)\left[\frac{(\alpha-1)}{2 \alpha R} i \frac{1}{k_{2}}+\frac{1}{16 R^{2}} \frac{3 \alpha^{2}+2 \alpha+3}{\alpha^{2}} \frac{1}{k_{2}^{2}}\right] e^{i k_{2}(1-\alpha) R}+c . c .
$$

Since $\alpha \neq 1$, we have that

$$
\frac{d}{d R}(1-\alpha) R \neq 0
$$


Therefore, $\beta_{[R, R]}^{-}$is of the form in Lemma 2 L.2.a in the domain of interest.

Since $\beta_{[R, R]}^{+}, \beta_{[R, R]}^{-}$and $\beta_{[r, R]}^{+}$are all of the form in Lemma 2 L.2.a, they are functions of rapid decrease in $k_{2}$. Then, from (4.22), we get,

$$
\beta=\beta_{[r, R]}^{-}+O\left(\frac{1}{k_{2}^{3}}\right) .
$$

A rough estimate of $\beta_{[r, R]}^{-}$from (4.19) gives $\beta_{[r, R]}^{-}=O(1)$. This implies that,

$$
|\beta|^{2}=\left|\beta_{[r, R]}^{-}\right|^{2}+O\left(\frac{1}{k_{2}^{3}}\right)
$$

A term of $O\left(1 / k_{2}^{3}\right)$ gives a convergent contribution to the integral in Eq.(2.10), and it only remains to estimate $\beta_{[r, R]}^{-}$with more care.

As in [11], we show (for a large class of embeddings $R(r)$ ) that a judicious use of the stationary phase approximation to estimate $\beta_{[r, R]}^{-}$obtains a divergent contribution to (2.10). Specifically, we demand that the embedding (and our choice of $\alpha_{0}$ ) be such that the following "genericity" conditions hold.

(a) There exists a number $\alpha_{0} \neq 1$ with the property that $R^{\prime}=\alpha_{0}$ at only finitely many points $r_{I}, I=1, \ldots, N$ in $[c, d]$.

(b) $R^{\prime \prime} \neq 0$ at $r_{I}$.

(c) At least one of $\tilde{\tilde{f}}_{I}, I=1, \ldots, \tilde{\tilde{N}}$, (see(4.33) below) is non-zero.

Condition (a) implies that there exists a fixed finite number of critical points for the exponent in (4.19) at $\alpha=\alpha_{0}$. Note that the condition for critical points is $R^{\prime}=\alpha$. This condition defines a map ' $\lambda$ ' from $r \in \Sigma$ to $\alpha \in\left(\alpha_{0}-\delta, \alpha_{0}+\delta\right)$. From this point of view, (b) states that the differential of this map is non-degenerate at $r=r_{I}\left(\right.$ with $\left.\lambda\left(r_{I}\right)=\alpha_{0}\right)$. An application of the inverse mapping theorem to $\lambda$ implies that the number of critical points $N$ does not change as $\alpha$ varies in a sufficiently small neighborhood of $\alpha_{0}$ (i.e. if $\delta$ is chosen small enough) and that the location of the critical points varies continuously with $\alpha$ in this neighborhood.

After these preliminaries, we proceed with our estimate for $\beta_{[r, R]}^{-}$. The leading order term in (4.19) is,

$$
\frac{1}{4 \pi \alpha^{1 / 2}} \int_{\bar{c}}^{\bar{d}} d R g(R)\left(\frac{r-R}{\sqrt{r R}}\right) e^{-i k_{2}(\alpha r-R)}+\text { c.c. . }
$$

We apply the stationary phase approximation to estimate (4.28). As discussed above, there are $N$ critical points $r_{I}$ where $R^{\prime}=\alpha$ and we estimate (4.28) as

$$
\frac{1}{4 \pi \alpha^{1 / 2}}\left(\frac{2 \pi}{k_{2}}\right)^{1 / 2} \sum_{I=1}^{N} f_{I} e^{-i k_{2} G_{I}}+\text { c.c. }+O\left(\frac{1}{k_{2}^{3 / 2}}\right),
$$

where

$$
\begin{aligned}
f_{I} & :=\left|\operatorname{det} R^{\prime \prime}\left(r_{I}\right)\right|^{-1 / 2}\left(\frac{r_{I}-R\left(r_{I}\right)}{\sqrt{r_{I} R\left(r_{I}\right)}}\right) e^{i \pi \operatorname{sign} R^{\prime \prime} / 4} \\
G_{I} & :=\alpha r_{I}-R\left(r_{I}\right)
\end{aligned}
$$


Note that $r_{I}$ depend on $\alpha$, i.e. $r_{I}=r_{I}(\alpha)$.

The stationary phase approximation applied to the subleading order terms in (4.19) yields an estimate of $O\left(1 / k_{2}^{3 / 2}\right)$, which is the same order as the "error term" in Eq.(4.29). Then, we have

$$
\beta_{[r, R]}^{-}=\frac{1}{4 \pi \alpha^{1 / 2}}\left(\frac{2 \pi}{k_{2}}\right)^{1 / 2} \sum_{I=1}^{2 N} \tilde{f}_{I} e^{-i k_{2} \tilde{G}_{I}}+O\left(\frac{1}{k_{2}^{3 / 2}}\right),
$$

where, for $I=1,2, \ldots, N, \tilde{f}_{I}=f_{I}, \tilde{G}_{I}=G_{I}, \tilde{f}_{I+N}=f_{I}^{*}, \tilde{G}_{I+N}=-G_{I}$, with $f_{1}, \ldots, f_{N}$ and $G_{1}, \ldots, G_{N}$ defined in Eqs.(4.30) and (4.31). It is possible that $\tilde{G}_{I}=\tilde{G}_{J}$ for some $I \neq J$, $I, J=1,2, \ldots, 2 N$. By suitably redefining the $\tilde{f}_{I}$, with the redefined $\tilde{f}_{I}$ denoted by $\tilde{\tilde{f}}_{I}$, the sum $\sum_{I=1}^{2 N} \tilde{f}_{I} e^{-i k_{2} \tilde{G}_{I}}$ can be rewritten as a sum over a finite number $\tilde{\tilde{N}}<2 N$ of terms, each of the form $\tilde{\tilde{f}}_{I} e^{-i k_{2} \tilde{\tilde{G}}_{I}}, I=1,2, \ldots, \tilde{\tilde{N}}, \tilde{\tilde{G}}_{I} \neq \tilde{\tilde{G}}_{J}$ for $I \neq J$. Then, we get,

$$
\left|\beta_{[r, R]}^{-}\right|^{2}>\frac{1}{8 \pi k_{2} \alpha}\left(\sum_{I}^{\tilde{\tilde{N}}}\left|\tilde{\tilde{f}}_{I}\right|^{2}+\sum_{I \neq J}^{\tilde{\tilde{N}}} \tilde{\tilde{f}}_{I} \tilde{\tilde{f}}_{J}^{*} e^{-i k_{2}\left(\tilde{\tilde{G}}_{I}-\tilde{\tilde{G}}_{n}\right)}\right)+O\left(\frac{1}{k_{2}^{2}}\right)
$$

For generic embeddings, at least one of the $\tilde{\tilde{f}}_{I} \neq 0$ and as mentioned in (c) above, we restrict our attention to such embeddings.

Now, we are ready to estimate the integral in Eq.(2.10) in the domain of interest. Using Eqs.(4.27) and (4.33) the integral is,

$$
\begin{aligned}
\int_{k_{2(0)}}^{2 k_{2(0)}} k_{2} d k_{2} \int_{\alpha_{0}-\delta}^{\alpha_{0}+\delta} d \alpha\left|\beta\left(k_{1}, k_{2}\right)\right|^{2} \\
>\frac{1}{2} \int_{k_{2(0)}}^{2 k_{2(0)}} k_{2} d k_{2} \int_{\alpha_{0}-\delta}^{\alpha_{0}+\delta} d \alpha\left|\beta_{[r, R]}^{-}\right|^{2} \\
>\frac{1}{16 \pi} \int_{k_{2(0)}}^{2 k_{2(0)}} d k_{2} \int_{\alpha_{0}-\delta}^{\alpha_{0}+\delta} \frac{d \alpha}{\alpha} \sum_{I}\left|\tilde{\tilde{f}}_{I}\right|^{2} \\
-\left|\frac{1}{16 \pi} \int_{k_{2(0)}}^{2 k_{2(0)}} d k_{2} \int_{\alpha_{0}-\delta}^{\alpha_{0}+\delta} \frac{d \alpha}{\alpha} \sum_{I \neq J}^{\frac{\tilde{N}}{\tilde{f}}} \tilde{\tilde{f}}_{I} \tilde{\tilde{f}}_{J}^{*} e^{-i k_{2}\left(\tilde{\tilde{G}}_{I}-\tilde{\tilde{G}}_{n}\right)}\right| \\
-\left|\frac{1}{16 \pi} \int_{k_{2(0)}}^{2 k_{2(0)}} d k_{2} \int_{\alpha_{0}-\delta}^{\alpha_{0}+\delta} \frac{d \alpha}{\alpha} \frac{C}{k_{2}}\right|,
\end{aligned}
$$

for some appropriate $C>0$. Since at least one $\tilde{\tilde{f}}_{I} \neq 0$, 4.36) is equal to $A k_{2(0)}$ for some suitably defined positive constant $A$. (Note that $A$ depends on $\alpha_{0}$ and $\delta$ but is independent of $\left.k_{2(0)}\right)$. Eq.(4.37) is estimated as of $O(1)$ (in $\left.k_{2(0)}\right)$ by integrating over $k_{2}$ to get

$$
\left.\int_{\alpha_{0}-\delta}^{\alpha_{0}+\delta} \frac{d \alpha}{\alpha} \sum_{I \neq J}^{\tilde{\tilde{N}}} \frac{\tilde{\tilde{f}}_{I} \tilde{\tilde{f}}_{J}^{*}}{\left(\tilde{\tilde{G}}_{I}-\tilde{\tilde{G}}_{J}\right)} i e^{-i k_{2}\left(\tilde{\tilde{G}}_{I}-\tilde{\tilde{G}}_{J}\right)}\right|_{k_{2(0)}} ^{2 k_{2(0)}}=O(1)
$$


Similarly an integration over $k_{2}$ show that (4.38) is also of $O(1)$. All this implies that, for large enough $k_{2(0)}$

$$
\int_{k_{2(0)}}^{2 k_{2(0)}} k_{2} d k_{2} \int_{\alpha_{0}-\delta}^{\alpha_{0}+\delta} d \alpha\left|\beta\left(k_{1}, k_{2}\right)\right|^{2}>\frac{A}{2} k_{2(0)}
$$

which diverges as $k_{2(0)} \rightarrow \infty$. Thus, we have shown that the contribution to (2.10) from the domain of interest is (linearly) divergent. Therefore, the action of a generic spatial diffeomorphism is not of Hilbert-Schmidt type.

\section{NONEXISTENCE OF INFINITESIMAL GENERATORS OF SPATIAL DIF- FEOMORPHISM}

After our analysis of finite spatial diffeomorphisms in the previous section, we now turn to a study of infinitesimal diffeomorphisms. Specifically, we are interested in investigating if the quantum operators corresponding to generators of finite spatial diffeomorphisms are densely defined on the dense set consisting of the Fock vacuum and suitably defined Nparticle states. Preliminary computations along the lines of [5] suggest that the generator of a spatial diffeomorphism is well-defined on this dense domain iff its action on the Fock vacuum is well-defined. Hence, we investigate if the action of such a generator is well-defined on the Fock vacuum.

Note that this section constitutes a digression from the main theme of this paper in that there is no direct relation between the existence (or lack thereof) of the action of such a generator on the Fock vacuum and the lack of unitary implementability of generic finite spatial diffeomorphisms. In particular, even if the generator turned out to be a densely-defined self-adjoint operator, it would not necessarily imply the existence of a unitary operator corresponding to the finite diffeomorphisms it (putatively) generates because there could be operator domain problems in defining ("path-ordered") exponentiation. Conversely, had finite diffeomorphisms been unitarily implementable, this would not necessarily imply that the infinitesimal generators were defined as self-adjoint operators or, even if they were, that the vacuum was in their dense domain.

Hence, our purpose is merely to initiate an exploration of properties of the generator of spatial diffeomorphisms in the hope that such an exploration may be useful for further work in the field. The result of our computations is that operators corresponding to generic generators of spatial diffeomorphisms ${ }^{7}$ of the flat initial slice $\Sigma_{0}$ do not have a well-defined action on the Fock vacuum. The generator of spatial diffeomorphisms of a slice $\Sigma$ in classical theory is $\int_{\Sigma} d r f(r) \pi \psi^{\prime}$ where $f(r)$ is the radial shift vector. When $\Sigma=\Sigma_{0}$, a straightforward computation shows that the action of the corresponding normal ordered quantum operator on the Fock vacuum state is,

$$
\| \int_{0}^{\infty} d r r f(r): \widehat{\dot{\psi}(r, 0)} \widehat{\psi^{\prime}(r, 0)}:|0\rangle \|^{2}=\frac{1}{8} \int_{0}^{\infty} d k_{1} \int_{0}^{\infty} d k_{2}\left|F\left(k_{1} k_{2}\right)\right|^{2},
$$

where

$$
F\left(k_{1}, k_{2}\right)=k_{1} k_{2} \int_{0}^{\infty} d r r f(r)\left[J_{0}\left(k_{1} r\right) J_{1}\left(k_{2} r\right)+J_{1}\left(k_{1} r\right) J_{0}\left(k_{2} r\right)\right]
$$

\footnotetext{
7 The notion of genericity used here is defined later in this section and in Appendix E We do not know how it relates to the genericity conditions of section IV.
} 
Here the radial shift, $f(r)$, is assumed to be of compact support away from $r=0$. We use Eq. (2.2) and the canonical commutation relations between the $\hat{a}(k)$ and $\hat{a}^{\dagger}(k)$ in the left hand side of Eq.(5.1) to get Eq.(15.2). If the integral on the right hand side of Eq.(15.1) diverges, the action of the generator is not well-defined. In the rest of the section we show that this is indeed the case.

To show that the integral in Eq.(5.1) diverges, we only need to show that it does so for a sub-domain of integration because the integrand is always positive. We restrict attention to the sub-domain $k_{2}>k_{1}, k_{2}-k_{1}=a$ with $a$ of $O(1)$ and $k_{1}, k_{2} \rightarrow \infty$. Using the Hankel transformation, $G(k)$, of $r f(r)$ with respect to $J_{0}(k r)$ (see Eq.(D1)) and identities in Appendix D, we get

$$
F\left(k_{1}, k_{2}\right)=k_{1} k_{2} \int_{k_{2}-k_{1}}^{k_{2}+k_{1}} d k k G(k)\left[\frac{\theta\left(k_{1}, k ; k_{2}\right)}{\pi k_{2}}+\frac{\theta\left(k_{2}, k ; k_{1}\right)}{\pi k_{1}}\right]+k_{1} \int_{0}^{k_{2}-k_{1}} d k k G(k),
$$

where $\theta\left(k_{1}, k ; k_{2}\right)$ is the angle between $k_{1}$ and $k$ in the triangle formed by $k_{1}, k$ and $k_{2}$, and similarly for $\theta\left(k_{2}, k ; k_{1}\right)^{8}$. By adding and subtracting terms, and using $\theta\left(k_{2}, k ; k_{1}\right)+$ $\theta\left(k_{1}, k ; k_{2}\right)+\theta\left(k_{2}, k_{1} ; k\right)=\pi$, we get

$$
\begin{aligned}
F\left(k_{1}, k_{2}\right) & =k_{1} \int_{0}^{k_{1}+k_{2}} d k k G(k) \\
& -k_{1} \int_{k_{2}-k_{1}}^{k_{2}+k_{1}} d k k G(k) \frac{\theta\left(k_{2}, k_{1} ; k\right)}{\pi} \\
& +\int_{k_{2}-k_{1}}^{k_{2}+k_{1}} d k k G(k)\left(k_{2}-k_{1}\right) \frac{\theta\left(k_{2}, k ; k_{1}\right)}{\pi} .
\end{aligned}
$$

Before we jump into the rather lengthy estimate of each term, it would be helpful to state the main results here. It turns out that, for sufficiently generic embeddings, the leading contribution to $F\left(k_{1}, k_{2}\right)$ comes from (5.5) in the domain we are interested in. Using the estimate of (5.5) we show that the right hand side of Eq.(5.1) diverges. Contributions from both (5.4) and (5.6) turn out to be negligible compared to the one from (5.5).

First, we estimate the integral in Eq.(5.4). It can be written as

$$
k_{1} \int_{0}^{k_{1}+k_{2}} d k k G(k)=k_{1} \int_{0}^{\infty} d k k G(k)-k_{1} \int_{k_{1}+k_{2}}^{\infty} d k k G(k) .
$$

The first integral on the right hand side is zero because, from Eq.(D2), it is equal to $\left.r f(r)\right|_{r=0}$, and $f(r)$ does not have support at $r=0^{9}$. Moreover, as shown in Appendix A.1 of [5], $G(k)$ falls faster than any power of $1 / k$ as $k \rightarrow \infty$. Since $k>\left(k_{1}+k_{2}\right), k \rightarrow \infty$, and the contribution from the second integral is negligible.

Next, consider Eq.(5.5). There are two contributions, namely, from $G(k)$ and from $\theta\left(k_{1}, k_{2} ; k\right)$. Since $G(k)$ falls off rapidly when $k \rightarrow \infty$, and $\theta\left(k_{1}, k_{2} ; k\right)$ is bounded, large

\footnotetext{
8 The notation used here is following. $\theta\left(k_{a}, k_{b} ; k_{c}\right)$ is defined as the angle between $k_{a}, k_{b}$ in the triangle formed by $k_{a}, k_{b}$ and $k_{c}$.

9 The Hankel transformation with respect to $J_{0}(k r)$ can be thought of as a two dimensional Fourier transformation. Since $f(r)$ is Schwartz, so is its Fourier transform, $G(k)$. See Appendix A.1 of [5].
} 
contributions can arise only when $k$ is small. So, we need to estimate $\theta\left(k_{1}, k_{2} ; k\right)$ when $k$ is small.

Since $k_{1}, k_{2}$ and $k$ form a triangle, we have in the domain of interest that

$$
\begin{aligned}
k^{2} & =\left(k_{1}-k_{2}\right)^{2}+4 k_{1} k_{2} \sin ^{2} \frac{\theta}{2} \\
& =a^{2}+4 k_{1} k_{2} \sin ^{2} \frac{\theta}{2} .
\end{aligned}
$$

Since $0<\theta<\pi, k$ increases when $\theta$ increases, for fixed $k_{1}, k_{2}$. Therefore, $k$ is small when $\theta$ is small. For small $\theta$ we can expand $\sin \frac{\theta}{2}$ in Eq.(5.8) to get

$$
k^{2}=a^{2}+4 k_{1} k_{2}\left[\frac{\theta^{2}}{4}-\frac{\theta^{4}}{48}+O\left(\theta^{6}\right)\right] .
$$

Now, to see how small $\theta$ and $k$ should be to have a non-trivial contribution, we divide the domain into two regions; $\theta \geq\left(k_{1} k_{2}\right)^{-1 / 2+\epsilon}$ and $\theta<\left(k_{1} k_{2}\right)^{-1 / 2+\epsilon}$ where $0<\epsilon \ll 1 / 2$. If $\theta=\left(k_{1} k_{2}\right)^{-1 / 2+\epsilon}$, then $k^{2}=\left(k_{1} k_{2}\right)^{2 \epsilon}+O(1)$. Thus, $k \rightarrow \infty$ when $k_{1}, k_{2} \rightarrow \infty$. Since $k$ increases with $\theta$, the contribution from $\theta \geq\left(k_{1} k_{2}\right)^{-1 / 2+\epsilon}$ is negligible due to the nice fall off behavior of $G(k)$ at large $k$.

Consider the contribution to Eq. (5.5.5) from

$$
a<k<\sqrt{a^{2}+\left(k_{1} k_{2}\right)^{2 \epsilon}} .
$$

This implies that $0<4 \sin ^{2} \theta / 2<\left(k_{1} k_{2}\right)^{-1+2 \epsilon}$ so that the upper bound of $\theta$ in this range exceeds $\left(k_{1} k_{2}\right)^{-1 / 2+\epsilon}$ by $O\left(\left(k_{1} k_{2}\right)^{-3 / 2+3 \epsilon}\right)$. Since the contribution from the entire range $\theta \geq$ $\left(k_{1} k_{2}\right)^{-1 / 2+\epsilon}$ is negligible, it makes no difference whether we estimate the contribution from $\theta \leq\left(k_{1} k_{2}\right)^{-1 / 2+\epsilon}$ or from Eq.(5.10) and we use the latter.

In this range, for large enough $k_{1}, k_{2}$, we have from Eq.(15.9) that

$$
\theta=\left(\frac{k^{2}-a^{2}}{k_{1} k_{2}}\right)^{1 / 2}+O\left(\frac{1}{\left(k_{1} k_{2}\right)^{3 / 2-3 \epsilon}}\right) .
$$

Then, Eq.(5.5) satisfies the bounds

$$
\begin{aligned}
\left|k_{1} \int_{k_{2}-k_{1}}^{k_{2}+k_{1}} d k k G(k) \frac{\theta\left(k_{2}, k_{1} ; k\right) \mid}{\pi}\right| & >\left|k_{1} \int_{a}^{\sqrt{a^{2}+\left(k_{1} k_{2}\right)^{2 \epsilon}}} d k k G(k) \frac{\theta\left(k_{2}, k_{1} ; k\right)}{\pi}\right| \\
& -\left|k_{1} \int_{\sqrt{a^{2}+\left(k_{1} k_{2}\right)^{2 \epsilon}}}^{k_{1}+k_{2}} d k k G(k) \frac{\theta\left(k_{2}, k_{1} ; k\right)}{\pi}\right| \\
& >\left|\frac{k_{1}}{\pi} \int_{a}^{\sqrt{a^{2}+\left(k_{1} k_{2}\right)^{2 \epsilon}}} d k k G(k)\left(\frac{k^{2}-a^{2}}{k_{1} k_{2}}\right)^{1 / 2}\right| \\
& -\left|\frac{k_{1}}{\pi} \int_{a}^{\sqrt{a^{2}+\left(k_{1} k_{2}\right)^{2 \epsilon}}} d k k G(k) O\left(\frac{1}{\left(k_{1} k_{2}\right)^{3 / 2-3 \epsilon}}\right)\right| \\
& -\left|k_{1} \int_{\sqrt{a^{2}+\left(k_{1} k_{2}\right)^{2 \epsilon}}}^{k_{1}+k_{2}} d k k G(k) \frac{\theta\left(k_{2}, k_{1} ; k\right)}{\pi}\right| \\
:=\left|I_{2}\right|-\left|I_{3}\right|-\left|I_{1}\right|, &
\end{aligned}
$$


where we have used (5.11). As discussed above, $\left|I_{1}\right|$ is negligible. On the other hand, we have that

$$
\begin{aligned}
\left|I_{3}\right| & =\frac{k_{1}}{\pi\left(k_{1} k_{2}\right)^{3 / 2}}\left|\int_{a}^{\sqrt{a^{2}+\left(k_{1} k_{2}\right)^{2 \epsilon}}} d k k G(k) O\left[\left(k_{1} k_{2}\right)^{3 \epsilon}\right]\right| \\
& <\frac{C_{1} k_{1}}{\pi\left(k_{1} k_{2}\right)^{3 / 2-3 \epsilon}} \int_{a}^{\sqrt{a^{2}+\left(k_{1} k_{2}\right)^{2 \epsilon}}} d k k|G(k)| \\
& <\frac{C_{1} k_{1}}{\pi\left(k_{1} k_{2}\right)^{3 / 2-3 \epsilon}} \int_{0}^{\infty} d k k|G(k)| \\
& =\frac{k_{1}}{\left(k_{1} k_{2}\right)^{3 / 2-3 \epsilon}} C_{2},
\end{aligned}
$$

for appropriate constant $C_{1}$ and $C_{2}$. Finally $\left|I_{2}\right|$ is,

$$
\begin{aligned}
\left|I_{2}\right| & =\frac{k_{1}}{\pi}\left|\int_{a}^{\sqrt{a^{2}+\left(k_{1} k_{2}\right)^{2 \epsilon}}} d k k G(k)\left(\frac{k^{2}-a^{2}}{k_{1} k_{2}}\right)^{1 / 2}\right| \\
& =\frac{1}{\pi} \sqrt{\frac{k_{1}}{k_{2}}}\left|\int_{a}^{\sqrt{a^{2}+\left(k_{1} k_{2}\right)^{2 \epsilon}}} d k k G(k) \sqrt{k^{2}-a^{2}}\right| \\
& :=\frac{1}{\pi} \sqrt{\frac{k_{1}}{k_{2}}}\left|C\left(a, k_{1}, k_{2}\right)\right| .
\end{aligned}
$$

If $C\left(a, k_{1}, k_{2}\right) \neq 0$ as $k_{1}, k_{2} \rightarrow \infty$ it follows that $I_{2}$ dominates over $I_{3}$ in this limit. The leading contribution to (5.5) is then (5.14). We now show that, for sufficiently generic shift vector $f(r)$ (see Appendix $\mathbb{E}$ for the definition of genericity), this is indeed the case. The idea is to estimate $C\left(a, k_{1}, k_{2}\right)$ as $a \rightarrow 0$ and $k_{1}, k_{2} \rightarrow \infty$. Consider the behavior of $C\left(a, k_{1}, k_{2}\right)$ in the region where $a \in\left[\frac{1}{k_{2}^{\delta}}, \frac{2}{k_{2}^{\delta}}\right], 0<\delta \ll 1$ and $k_{1}, k_{2} \rightarrow \infty$. Define $\sigma$ as $\sigma:=a k_{2}^{\delta}$. Then,

$$
C\left(a, k_{1}, k_{2}\right)=\int_{\frac{\sigma}{k_{2}^{\delta}}}^{\left(k_{1} k_{2}\right)^{\epsilon}} d k k G(k) \sqrt{k^{2}-\left(\frac{\sigma}{k_{2}^{\delta}}\right)^{2}}+\int_{\left(k_{1} k_{2}\right)^{\epsilon}}^{\sqrt{\left(\sigma / k_{2}^{\delta}\right)^{2}+\left(k_{1} k_{2}\right)^{2 \epsilon}}} d k k G(k) \sqrt{k^{2}-\left(\frac{\sigma}{k_{2}^{\delta}}\right)^{2}} .
$$

The second integral in Eq.(5.15) is negligible since $G(k)$ falls faster than any power of $1 / k[5]$. 
The first integral in Eq.(5.15) can be estimated as,

$$
\begin{aligned}
\int_{\frac{\sigma}{k_{2}^{\delta}}}^{\left(k_{1} k_{2}\right)^{\epsilon}} d k \quad k G(k) \sqrt{k^{2}-\left(\frac{\sigma}{k_{2}^{\delta}}\right)^{2}} \\
=\int_{\frac{\sigma}{k_{2}^{\delta}}}^{\frac{\sigma}{k_{2}^{\delta / 2}}} d k k G(k) \sqrt{k^{2}-\left(\frac{\sigma}{k_{2}^{\delta}}\right)^{2}}+\int_{\frac{\sigma}{k_{2}^{\delta / 2}}}^{\left(k_{1} k_{2}\right)^{\epsilon}} d k k G(k) \sqrt{k^{2}-\left(\frac{\sigma}{k_{2}^{\delta}}\right)^{2}} \\
=O\left(\frac{1}{k_{2}^{\delta / 2}}\right)+\int_{\frac{\sigma}{k_{2}^{\delta / 2}}}^{\left(k_{1} k_{2}\right)^{\epsilon}} d k k^{2} G(k)+\int_{\frac{\sigma}{k_{2}^{\delta / 2}}}^{\left(k_{1} k_{2}\right)^{\epsilon}} d k k^{2} G(k) O\left(\frac{1}{k_{2}^{\delta}}\right) \\
=O\left(\frac{1}{k_{2}^{\delta / 2}}\right)+\int_{\frac{\sigma}{k_{2}^{\delta / 2}}}^{\left(k_{1} k_{2}\right)^{\epsilon}} d k k^{2} G(k)+O\left(\frac{1}{k_{2}^{\delta}}\right) \\
=O\left(\frac{1}{k_{2}^{\delta / 2}}\right)+\int_{\frac{\sigma}{k_{2}^{\delta / 2}}}^{\left(k_{1} k_{2}\right)^{\epsilon}} d k k^{2} G(k) .
\end{aligned}
$$

In the second line, we divide the domain of integration because we want to expand $\sqrt{k^{2}-\left(\frac{\sigma}{k_{2}^{\delta}}\right)^{2}}$. This is possible only when $k \gg \sigma / k_{2}^{\delta}$, i.e. in the second term. Now,

$$
\int_{\frac{\sigma}{k_{2}^{\delta / 2}}}^{\left(k_{1} k_{2}\right)^{\epsilon}} d k k^{2} G(k)=\int_{0}^{\infty} d k k^{2} G(k)-\int_{0}^{\frac{\sigma}{k_{2}^{\delta / 2}}} d k k^{2} G(k)-\int_{\left(k_{1} k_{2}\right)^{\epsilon}}^{\infty} d k k^{2} G(k) .
$$

The second integral in Eq.(5.17) is of $O\left(\frac{\sigma}{k^{\delta / 2}}\right)$, while the third integral can be neglected since $G(k)$ falls faster than any power of $1 / k,[5]$. This implies that, for a suitable constant $E$,

$$
\begin{aligned}
\left|C\left(a, k_{1}, k_{2}\right)\right| & >\left|\int_{0}^{\infty} d k k^{2} G(k)\right|-\frac{E \sigma}{k_{2}^{\delta / 2}} \\
& >\frac{1}{2}\left|\int_{0}^{\infty} d k k^{2} G(k)\right|
\end{aligned}
$$

provided $\int_{0}^{\infty} d k k^{2} G(k) \neq 0$. In Appendix [E, we show that this is indeed the case for generic $G(k)$. Hence, we conclude that the estimate of Eq.(5.5) is,

$$
\begin{aligned}
\left|k_{1} \int_{k_{2}-k_{1}}^{k_{2}+k_{1}} d k k G(k) \frac{\theta\left(k_{2}, k_{1} ; k\right)}{\pi}\right| & >\frac{1}{2 \pi} \sqrt{\frac{k_{1}}{k_{2}}}\left|\int_{0}^{\infty} d k k^{2} G(k)\right|-\frac{k_{1}}{\left(k_{1} k_{2}\right)^{3 / 2-3 \epsilon} D} \\
& >\frac{1}{4 \pi} \sqrt{\frac{k_{1}}{k_{2}}}\left|\int_{0}^{\infty} d k k^{2} G(k)\right|
\end{aligned}
$$

for sufficiently large $k_{1}, k_{2}$.

Before we estimate $F\left(k_{1}, k_{2}\right)$, we need show that Eq.(5.6) is negligible compared to 
Eq.(5.5). For the range of variables we consider, Eq.(5.6) satisfies,

$$
\begin{aligned}
\left|\int_{k_{2}-k_{1}}^{k_{2}+k_{1}} d k k G(k)\left(k_{2}-k_{1}\right) \frac{\theta\left(k_{2}, k ; k_{1}\right)}{\pi}\right| & <a \int_{a}^{k_{1}+k_{2}} d k|k G(k)| \\
& <a \int_{0}^{\infty} d k|k G(k)| \\
& =O\left(\frac{1}{k_{2}^{\delta}}\right) .
\end{aligned}
$$

where we used the fact $0<\theta<\pi$ in the first line.

Now, we are ready to estimate $F\left(k_{1}, k_{2}\right)$. From the Eqs.(5.7), (5.13), (5.14) and (5.18),

$$
\begin{aligned}
\left|F\left(k_{1}, k_{2}\right)\right| & >\frac{1}{8 \pi} \sqrt{\frac{k_{1}}{k_{2}}}\left|\int_{0}^{\infty} d k k^{2} G(k)\right| \\
& >\frac{1}{16 \pi}\left|\int_{0}^{\infty} d k k^{2} G(k)\right| \\
& :=C_{0},
\end{aligned}
$$

for sufficiently large $k_{1}, k_{2}$ and $k_{2}-k_{1} \in\left[\frac{1}{k_{2}^{\delta}}, \frac{2}{k_{2}^{\delta}}\right]$.

Finally, we have all the necessary ingredients to estimate the integral in Eq.(5.1) in the range of interest. First, we change variables from $\left(k_{1}, k_{2}\right)$ to $\left(a, k_{2}\right)$, where $a=k_{2}-k_{1}$ as before. Then, we integrate $|F|^{2}$ over the domain $a \in\left[\frac{1}{k_{2}^{\delta}}, \frac{2}{k_{2}^{\delta}}\right]$, and $k_{2} \in\left[\left(k_{2}\right)_{0}, 2\left(k_{2}\right)_{0}\right] ;\left(k_{2}\right)_{0} \rightarrow$ $\infty$. The integral in Eq.(5.1) is, then,

$$
\begin{aligned}
\frac{1}{2} \int_{0}^{\infty} d k_{1} \int_{0}^{\infty} d k_{2}\left|F\left(k_{1}, k_{2}\right)\right|^{2} & >\frac{1}{2} \int_{\left(k_{2}\right)_{0}}^{2\left(k_{2}\right)_{0}} d k_{2} \int_{1 / k_{2}^{\delta}}^{2 / k_{2}^{\delta}} d a\left|F\left(k_{1}, k_{2}\right)\right|^{2} \\
& >\frac{1}{2} \int_{\left(k_{2}\right)_{0}}^{2\left(k_{2}\right)_{0}} d k_{2} \int_{1 / k_{2}^{\delta}}^{2 / k_{2}^{\delta}} d a\left(C_{0}\right)^{2} \\
& =\frac{1}{2} \frac{\left(C_{0}\right)^{2}}{1-\delta}\left(k_{2}\right)_{0}^{1-\delta}\left[2^{1-\delta}-1^{1-\delta}\right] \rightarrow \infty
\end{aligned}
$$

for small $\delta$. Therefore, the action of the normal-ordered generator of generic spatial diffeomorphisms of $\Sigma_{0}$ on the Fock vacuum is not well-defined.

\section{DISCUSSION}

The work of Kuchař [3] maps the dynamics of cylindrical waves to that of an axisymmetric free scalar field along arbitrary axisymmetric foliations of the fixed $(2+1)$-dimensional flat spacetime (1.2). In this work we studied quantum evolution of this free field operator from the initial flat $T=0$ slice, $\Sigma_{0}$, with radial coordinate $R$, to an arbitrary slice $\Sigma$ with radial coordinate $r$. We showed that operator evolution is unitarily implemented in the standard Fock representation when $r$ is chosen to coincide with $R$ on $\Sigma$. The transition to a general radial coordinate $r(R)$ from $R$ is obtained by the action of a corresponding spatial diffeomorphism on the field variables on $\Sigma$. We showed that for generic choices of $r(R)$, this diffeomorphism is not unitarily implemented in the standard Fock space representation. 
In $(1+1)$-dimensional parametrized field theory (PFT) where such operator evolution is unitary, a Tomonaga-Schwinger type of functional evolution can be defined as the unitary evolution of Fock states from the initial $T=0$ surface to an arbitrary one [10]. Our results in this work indicate that a similar notion of functional evolution for quantum cylindrical waves is not a viable concept for generic choices of foliations starting from the slice $\Sigma_{0}$. One may inquire if such functional evolution can be defined in the standard Fock representation by choosing some other slice than $\Sigma_{0}$ as the initial slice (this would entail expressing the standard mode coefficients $a(k)$ as functionals of data on the new initial slice and analysing the Bogolubov transformation corresponding to evolution of these mode coefficients). ${ }^{10}$ Though we have not investigated this question, we suspect that no such choice of initial slice can render generic evolution of operators as unitary.

Our results do indicate, however, that it should be possible to define functional evolution of quantum states along the restricted class of foliations wherein the radial coordinate on each slice is $R$. Such evolution is formally described by the 'half parametrized' formalism of 3]. It would be of interest to construct the Schrödinger picture states as the unitary image of the Heisenberg-picture Fock states and to show that they satisfy a functional Schrödinger equation along the lines of [10], for this restricted evolution.

Our results have been obtained for foliations satisfying the boundary conditions (2.11) and (2.12). The fact that $T^{\prime}(r)$ and $R(r)-r$ vanish at the axis and at spatial infinity are direct consequences of the boundary conditions for cylindrical waves in their ADM description (see the appendix of [16]). Our conditions of compact support for these quantities are more restrictive than the conditions in [16]. It would be of interest to seek, either a generalization of our proofs to those boundary conditions, or an alternative set of boundary conditions which allows the definition of a consistent Hamiltonian framework and which induce conditions on $T(r), R(r)$ such that our proofs still go through. We suspect that requiring fall offs of various phase space variables to be faster than any power of $r^{-1}$ at spatial infinity and any power of $r$ at the axis should suffice for the latter. It would be good to check this.

In section $\mathrm{V}$ we showed that a generic infinitesimal generator of spatial diffeomorphisms on $\Sigma_{0}$ does not have a well defined action on the Fock vacuum. What about generators of infinitesimal 'half parametrized' evolution on $\Sigma_{0}$ ? From [3] the relevant Hamiltonian density on any slice $\Sigma$ with radial coordinate $R$ is given by

$$
\mathcal{H}=\frac{1}{2}\left(1-T_{, R}^{2}\right)^{-1}\left[\left(R^{-\frac{1}{2}} \pi-R^{\frac{1}{2}} T_{, R} \psi_{, R}\right)^{2}+\frac{1}{2} R \psi_{, R}^{2}\right] .
$$

On $\Sigma_{0}, T_{, R}=0, \pi=R \psi_{, T}$ and $\mathcal{H}$ equals the standard flat spacetime energy density. $H(f):=\int d R f(R) \mathcal{H}$ is a generator of 'half parametrized' evolution on $\Sigma_{0}$. In [5] it was shown that for smooth $f$ vanishing fast enough at the axis and at spatial infinity, the normal-ordered operator $\hat{H}(f)$ is densely defined and has a well-defined action on the Fock vacuum. Since we know that the standard flat space Hamiltonian, $\hat{H}_{0}$, is also a well-defined operator, we conclude from [5] that $\hat{H}(f+1)=\hat{H}(f)+\hat{H}_{0}$ which generates nontrivial evolution at infinity and along the axis is also well-defined.

10 Note that an affirmative answer for the existence of generic evolution from the new initial slice would be consistent with our considerations here, only if the specific evolution between the new initial slice and $\Sigma_{0}$ was non-unitary. 
It can be checked that the Poisson bracket between two such generators of 'half parametrized' evolution on $\Sigma_{0}, H\left(f_{1}\right)$ and $H\left(f_{2}\right)$ is the generator of a spatial diffeomorphism. It seems puzzling that despite $\hat{H}\left(f_{1}\right), \hat{H}\left(f_{2}\right)$ being densely defined operators, the generators of generic spatial diffeomorphisms do not have a well-defined action on the Fock vacuum. A possible resolution is that the relevant shift vector does not satisfy our requirements of genericity since it is built out of $f_{1}, f_{2}$ in a specific way. However, we suspect that the resolution lies in the well-known problem of domains for unbounded operators (see for e.g. 17]): although the vacuum is in the domain of both $\hat{H}\left(f_{1}\right)$ and $\hat{H}\left(f_{2}\right)$, it need not be so for their product and/or commutator. It would be of interest to confirm this.

Next, we comment on the relevance of the recent work of Corichi, Cortez, and Mena Marugán [18] to our considerations here. They considered the issue of unitary implementability of evolution in the context of a quatization of the Gowdy model. The dynamics of the Gowdy model is also determined by that of a scalar field on an auxilliary $2+1$ dimensional spacetime. Pierri [19] showed that the scalar field admits a natural quantization based on the choice of a specific foliation of the auxilliary spacetime. Corichi et al. [20] and Torre 21] showed that in the context of this fixed foliation and Pierri's quantization, scalar field evolution is not unitarily implemented. However, in 18], a quantization was constructed in which the evolution of a time-dependent rescaling of the scalar field is unitary. As far as we can tell, this quantization is inequivalent to that of [19]. In view of the results of [18], the following questions are of interest in the context of our work here. Given an arbitrary but fixed axisymmetric foliation of $2+1$ dimensional flat spacetime, is there a quantization in which a suitably redefined scalar field variable evolves unitarily? If so, is this quantization equivalent to the standard Fock quantization?

We conclude with some remarks regarding the relevance of our results to a Dirac quantization of PFT and of gravity. At first glance, our results seem to indicate that a straightforward Dirac quantization of axisymmetric PFT in $2+1$ dimensions (and hence of quantum cylindrical waves) which is equivalent to the standard Fock quantization, does not exist. 11 Indeed it seems that the lack of unitary implementability of spatial diffeomorphisms for quantum cylindrical waves has an adverse lesson for Loop Quantum Gravity (see for example [22]) since the entire formalism is based on a unitary representation of spatial diffeomorphisms. However, a more careful Loop Quantum Gravity type treatment of PFT merits the construction of a kinematic Hilbert space representation for both the matter and the embedding variables rather than formally setting the action of $\hat{X}^{\alpha}(x)$ to be multiplication by the embedding variable $X^{\alpha}(x)$ and that of the conjugate momentum variable to be $\hat{P}_{\alpha}(x)=-i \frac{\delta}{\delta X^{\alpha}(x)}$. Such a treatment is currently in progress [23] and suggests that when the embedding sector is properly defined in the quantum theory along with an appropriate notion of embedding dependent Hilbert spaces for the matter sector, a satisfactory Dirac quantization which is equivalent to the standard Fock quantization can be constructed notwithstanding the results of this work and [1]].

11 Note that a classical Hamilton- Jacobi type of canonical transformation along the lines of [9] should result in the classical Heisenberg picture constraints which directly yield, upon Dirac quantization, the standard Fock space. However the PFT constraints which are naturally available take the form appropriate to the classical Schrödinger picture [9, 12]. Our results imply that a direct Dirac quantization of the latter, without further canonical transformations, is not equivalent to the standard Fock representation. 


\section{Acknowledgments}

We thank Charles Torre for useful comments on this work. We also thank an anonymous refree for going through the manuscript with great care.

\section{APPENDIX A: LEMMAS}

Lemma 1 Consider an integral

$$
I(p, q)=\int_{a}^{b} \operatorname{drg}(r) f_{1}(p r) e^{i q f_{2}(r)} e^{i p f_{3}(r)}
$$

that satisfies

- $p, q>0$.

- $g(r), f_{1}(r), f_{2}(r), f_{3}(r)$ are $C^{\infty}$.

- $g(r)$ has support only in $[a, b]$.

- $f_{2}^{\prime} \neq 0$ in $[a, b]$.

- $f_{1}$ and all its derivatives are bounded in $(0, \infty)$.

Then,

$$
|I|<\frac{p}{q} M_{1}+\frac{1}{q} M_{2},
$$

for suitable constants $M_{1}$ and $M_{2}$.

Proof : Let $f_{2}(r)=y$. Since $f_{2}^{\prime} \neq 0$, and $f$ is $C^{\infty}$ in $[a, b], f_{2}$ has a $C^{\infty}$-inverse in $[a, b]$, $\chi(y)=r$. Then,

$$
I(p, q)=\int_{\bar{a}}^{\bar{b}} d y\left(\frac{d \chi}{d y}\right) g[\chi(y)] f_{1}[p \chi(y)] e^{i p f_{3}[\chi(y)]} e^{i q y}
$$

where $\chi(\bar{a})=a$ and $\chi(\bar{b})=b$. After integration by parts we get Eq. (A2) because all functions and their derivatives in the integral are $C^{\infty}$ and bounded.

Lemma 2 - L.2.a : $\int_{a}^{b} d r g(r) e^{i k f(r)} \rightarrow 0$ faster than $\frac{1}{k^{n}}$ for any $n$ as $k \rightarrow \infty$, if $f^{\prime}(r) \neq 0$ in $[a, b], g(r)$ and $f(r)$ are $C^{\infty}$, and $g(r)$ has compact support in $[a, b]$.

- L.2.b : $\int_{a}^{b} d r g(r) e^{i k f(r)} \rightarrow 0$ as $\frac{1}{k}$ for $k \rightarrow \infty$, if $f^{\prime}(r) \neq 0$ in $[a, b], g(r)$ and $f(r)$ are $C^{\infty}$, but either $g(a)$ or $g(b)$ or both are non-zero.

Proof : Similar to Lemma 1]

\section{APPENDIX B: ASYMPTOTIC EXPANSION OF BESSEL FUNCTIONS}

From 24] p199 we have that for large $|z|$ and $|\arg z|<\pi$,

$$
\begin{aligned}
& J_{0}(z) \sim \sqrt{\frac{2}{\pi z}}\left[\cos \left(z-\frac{\pi}{4}\right)+\sin \left(z-\frac{\pi}{4}\right) \frac{1}{8 z}-\cos \left(z-\frac{\pi}{4}\right) \frac{9}{128 z^{2}}+O\left(\frac{1}{z^{3}}\right)\right] \\
& J_{1}(z) \sim \sqrt{\frac{2}{\pi z}}\left[\cos \left(z-\frac{3 \pi}{4}\right)-\sin \left(z-\frac{3 \pi}{4}\right) \frac{3}{8 z}+\cos \left(z-\frac{3 \pi}{4}\right) \frac{15}{128 z^{2}}+O\left(\frac{1}{z^{3}}\right)\right] .
\end{aligned}
$$




\section{APPENDIX C: DERIVATION OF EQ.(3.2) FROM EQ.(3.1)}

Consider an integral

$$
\begin{aligned}
I\left(k_{1}, k_{2}, k\right) & :=i k \int_{0}^{\infty} d r r J_{0}\left(k_{1} r\right) J_{1}\left(k_{2} r\right) T^{\prime} e^{i k T(r)} \\
& =\int_{0}^{\infty} d r r J_{0}\left(k_{1} r\right) J_{1}\left(k_{2} r\right) \frac{d}{d r}\left[e^{i k T(r)}-e^{i k T(\infty)}\right] \\
& =\int_{0}^{\infty} d r r\left[k_{1} J_{1}\left(k_{1} r\right) J_{1}\left(k_{2} r\right)-k_{2} J_{0}\left(k_{1} r\right) J_{0}\left(k_{2} r\right)\right]\left[e^{i k T(r)}-e^{i k T(\infty)}\right],
\end{aligned}
$$

where we use integration by parts and Bessel function identities, $\frac{d}{d z}\left[z^{n} J_{n}(z)\right]=z^{n} J_{n-1}(z)$, $\frac{d}{d z} J_{0}(z)=-J_{1}(z)$ in the third line. Then,

$$
\begin{aligned}
I\left(k_{1}, k_{2}, k\right) & +I\left(k_{2}, k_{1}, k\right) \\
& =\left(k_{1}+k_{2}\right) \int_{0}^{\infty} d r r\left[J_{1}\left(k_{1} r\right) J_{1}\left(k_{2} r\right)-J_{0}\left(k_{1} r\right) J_{0}\left(k_{2} r\right)\right] e^{i k T(r)} \\
& -\left(k_{1}+k_{2}\right) \int_{0}^{\infty} d r r\left[J_{1}\left(k_{1} r\right) J_{1}\left(k_{2} r\right)-J_{0}\left(k_{1} r\right) J_{0}\left(k_{2} r\right)\right] e^{i k T(\infty)} \\
& =\left(k_{1}+k_{2}\right) \int_{0}^{\infty} d r r\left[J_{1}\left(k_{1} r\right) J_{1}\left(k_{2} r\right)-J_{0}\left(k_{1} r\right) J_{0}\left(k_{2} r\right)\right] e^{i k T(r)} \\
& -\left(k_{1}+k_{2}\right) e^{i k T(\infty)}\left[\frac{\delta\left(k_{1}, k_{2}\right)}{k_{1}}-\frac{\delta\left(k_{1}, k_{2}\right)}{k_{1}}\right] \\
& =\left(k_{1}+k_{2}\right) \int_{0}^{\infty} d r r\left[J_{1}\left(k_{1} r\right) J_{1}\left(k_{2} r\right)-J_{0}\left(k_{1} r\right) J_{0}\left(k_{2} r\right)\right] e^{i k T(r)} .
\end{aligned}
$$

Here we use an orthogonality relation for Bessel functions,

$$
\int_{0}^{\infty} d r r J_{n}(k r) J_{n}(l r)=\frac{\delta(k, l)}{k},
$$

in the fifth line. Eq. (3.1) is then,

$$
\begin{aligned}
\beta\left(k_{1}, k_{2}\right) & =-\frac{k_{1}}{2\left(k_{1}+k_{2}\right)}\left[I\left(k_{1}, k_{2}, k_{2}\right)+I\left(k_{2}, k_{1}, k_{2}\right)\right] \\
& =-\frac{i}{2} \frac{k_{1} k_{2}}{\left(k_{1}+k_{2}\right)} \int_{a}^{b} d r r\left[J_{0}\left(k_{1} r\right) J_{1}\left(k_{2} r\right)+J_{1}\left(k_{1} r\right) J_{0}\left(k_{2} r\right)\right] T^{\prime} e^{i k_{2} T}
\end{aligned}
$$

which is Eq.(3.2). In the last line, we use the compactness of $T^{\prime}$, Eq.(2.11).

\section{APPENDIX D: DERIVATION OF EQ.(5.3)}

In this section we present the derivation of Eq.(15.3) . The Hankel transform $G(k)$ of $r f(r)$ with respect to $J_{0}(k r)$ is [See, [24] p453]

$$
G(k)=\int_{0}^{\infty} d r r r f(r) J_{0}(k r)
$$




$$
r f(r)=\int_{0}^{\infty} d k k G(k) J_{0}(k r)
$$

Using Eq.(D2) into Eq.(5.2) we get

$$
F\left(k_{1} k_{2}\right)=k_{1} k_{2} \int_{0}^{\infty} d k G(k) k \int_{0}^{\infty} d r\left[J_{0}(k r) J_{0}\left(k_{1} r\right) J_{1}\left(k_{2} r\right)+J_{0}(k r) J_{1}\left(k_{1} r\right) J_{0}\left(k_{2} r\right)\right] .
$$

From p411 of [24]

$$
\begin{aligned}
\int_{0}^{\infty} d r J_{0}(k r) J_{0}\left(k_{1} r\right) J_{1}\left(k_{2} r\right) & =\frac{\theta\left(k_{1}, k ; k_{2}\right)}{\pi k_{2}} & & \text { if }\left|k-k_{1}\right|<k_{2}<\left|k+k_{1}\right| \\
& =\frac{1}{k_{2}} & & \text { if } k_{2}>k+k_{1} \\
& =0 & & \text { otherwise }
\end{aligned}
$$

and

$$
\begin{aligned}
\int_{0}^{\infty} d r J_{0}(k r) J_{0}\left(k_{2} r\right) J_{1}\left(k_{1} r\right) & =\frac{\theta\left(k_{2}, k ; k_{1}\right)}{\pi k_{1}} & & \text { if }\left|k-k_{2}\right|<k_{1}<\left|k+k_{2}\right| \\
& =\frac{1}{k_{1}} & & \text { if } k_{1}>k+k_{2} \\
& =0 & & \text { otherwise } .
\end{aligned}
$$

Using Eq.(D4) and Eq.(D5) into Eq.(D3) we get Eq.(15.3).

\section{APPENDIX E: PROOF OF $\int_{0}^{\infty} d k k^{2} G(k) \neq 0$ FOR GENERIC $G(k)$}

Recall, from Eq.(D1) and Eq.(D2), that $G(k)$ is the Hankel transform of $G(r):=r f(r)$ with respect to $J_{0}(k r)$. Using an integral representation of $J_{0}(k r)$ and rotational symmetry of $G(r), G(\vec{k}):=G(k)$ can be shown to be a two-dimensional Fourier transform of $G(\vec{r}):=$ $G(r)[5]$. That is,

$$
G(\vec{k})=\frac{1}{2 \pi} \int d x^{2} G\left(x_{1}, x_{2}\right) e^{i k_{1} x_{1}+i k_{2} x_{2}}
$$

However we can also consider $G\left(k_{1}, k_{2}=0\right)$ as a one-dimensional Fourier transform of $g\left(x_{1}\right)$ as defined below,

$$
\begin{aligned}
G\left(k_{1}, k_{2}=0\right) & =\frac{1}{\sqrt{2 \pi}} \int_{-\infty}^{\infty} d x_{1} e^{i k_{1} x_{1}}\left[\frac{1}{\sqrt{2 \pi}} \int_{-\infty}^{\infty} d x_{2} G\left(x_{1}, x_{2}\right)\right] \\
& :=\frac{1}{\sqrt{2 \pi}} \int_{-\infty}^{\infty} d x_{1} e^{i k_{1} x_{1}} g\left(x_{1}\right) .
\end{aligned}
$$

Note that $g\left(x_{1}\right)$ exists and posses good fall-off and smoothness property inherited from $G\left(x_{1}, x_{2}\right)$. Then, $G\left(k_{1}, k_{2}=0\right):=g\left(k_{1}\right)$ satisfies,

$$
g\left(x_{1}\right)=\frac{1}{\sqrt{2 \pi}} \int_{-\infty}^{\infty} d k_{1} e^{-i k_{1} x_{1}} g\left(k_{1}\right)
$$


Now,

$$
\frac{d^{2} g\left(x_{1}\right)}{d x_{1}^{2}}=-\frac{1}{\sqrt{2 \pi}} \int_{-\infty}^{\infty} d k_{1} e^{i k_{1} x_{1}} k_{1}^{2} g\left(k_{1}\right)
$$

which implies

$$
\begin{aligned}
\left.\frac{d^{2} g\left(x_{1}\right)}{d x_{1}^{2}}\right|_{x_{1}=0} & =-\frac{1}{\sqrt{2 \pi}} \int_{-\infty}^{\infty} d k_{1} k_{1}^{2} g\left(k_{1}\right) \\
& =-\sqrt{\frac{2}{2 \pi}} \int_{0}^{\infty} d k_{1} k_{1}^{2} g\left(k_{1}\right) \\
& =-\sqrt{\frac{2}{2 \pi}} \int_{0}^{\infty} d k k^{2} G(k)
\end{aligned}
$$

Here we have used the fact that $G\left(k_{1}, k_{2}=0\right):=g\left(k_{1}\right)$, and $G(\vec{k})=G(\|\vec{k}\|)$ due to the rotational symmetry.

To prove $\int_{0}^{\infty} d k k^{2} G(k) \neq 0$ for generic $G(k)$ we only need to prove that $\left.\frac{d^{2} g\left(x_{1}\right)}{d x_{1}^{2}}\right|_{x_{1}=0} \neq 0$ generically. For fixed $x_{1}$, we change variables from $\left(x_{1}, x_{2}\right)$ to $\left(x_{1}, r\right) ; x_{2} \mapsto \sqrt{x_{1}^{2}+x_{2}^{2}}=r$. Then, from Eq.(E2) and rotational symmetry of $G(\vec{x})$,

$$
\begin{aligned}
g\left(x_{1}\right) & =\sqrt{\frac{2}{\pi}} \int_{0}^{\infty} d x_{2} G\left(x_{1}, x_{2}\right) \\
& =\sqrt{\frac{2}{\pi}} \int_{x_{1}}^{\infty} d r r \frac{G(r)}{\sqrt{r^{2}-x_{1}^{2}}} .
\end{aligned}
$$

Since $G(r)$ is a function with compact support away from $r=0$, there exists a constant $r_{0}$ such that $G(r)=0$ for $r<r_{0}$. Then, for $x_{1}<r_{0}$, we get,

$$
\sqrt{\frac{2}{\pi}} \int_{x_{1}}^{\infty} d r r \frac{G(r)}{\sqrt{r^{2}-x_{1}^{2}}}=\sqrt{\frac{2}{\pi}} \int_{r_{0}}^{\infty} d r r \frac{G(r)}{\sqrt{r^{2}-x_{1}^{2}}}
$$

That is, for $x_{1}<r_{0}$,

$$
\frac{d^{2} g\left(x_{1}\right)}{d x_{1}^{2}}=\sqrt{\frac{2}{\pi}} \int_{r_{0}}^{\infty} d r r G(r) \frac{d^{2}}{d x_{1}^{2}}\left(\frac{1}{\sqrt{r^{2}-x_{1}^{2}}}\right) .
$$

Since we are interested in the limit $x_{1} \rightarrow 0$, we can use Eq.(E8). Then,

$$
\begin{aligned}
\left.\frac{d^{2} g\left(x_{1}\right)}{d x_{1}^{2}}\right|_{x_{1}=0} & =\left.\sqrt{\frac{2}{\pi}} \int_{r_{0}}^{\infty} d r r G(r) \frac{d^{2}}{d x_{1}^{2}}\right|_{x_{1}=0}\left(\frac{1}{\sqrt{r^{2}-x_{1}^{2}}}\right) \\
& =\sqrt{\frac{2}{\pi}} \int_{r_{0}}^{\infty} d r \frac{G(r)}{r^{2}} \\
& =\sqrt{\frac{2}{\pi}} \int_{r_{0}}^{\infty} d r \frac{f(r)}{r} \\
& =\sqrt{\frac{2}{\pi}} \int_{0}^{\infty} d r \frac{f(r)}{r},
\end{aligned}
$$


where we use the fact $f(r)=0$ for $r<r_{0}$ in the last line. For generic embedding the integral in the last line does not vanish, therefore, $\int_{0}^{\infty} d k k^{2} G(k) \neq 0$.

[1] C.W. Misner, in Magic Without Magic: John Archibald Wheeler, edited by J. Klauder (Freeman: San Francisco, 1972).

[2] M. Bojowald, Phys.Rev.Lett. 865227 (2001), (preprint gr-qc/0102069).

[3] K.V. Kuchař, Phys. Rev. D 4955 (1971).

[4] A. Ashtekar and M. Pierri, J. Math. Phys. 376250 (1996), (preprint gr-qc/9606085).

[5] M. Varadarajan, Class. Quantum Grav. 17189 (2000), (preprint gr-qc/9910043).

[6] M. Allen, Class. Quantum Grav. 4149 (1987).

[7] G.J.F. Barbero, I. Garay and E.J.S. Villasenõr, Phys.Rev.Lett. 95050501 (2005), (preprint gr-qc/0506093).

[8] M.E. Angulo and G.A. Mena Marugán, Int.J.Mod.Phys. D 9669 (2000), (preprint gr-qc/0002056); G.J.F. Barbero, G.A. Mena Marugán, and E.J.S. Villasenõr, Phys.Rev. D 70044028 (2004), (preprint gr-qc/0406087); Int.J.Mod.Phys. D 131119 (2004), (preprint gr-qc/0402096); Phys.Rev. D 67124006 (2003), (preprint gr-qc/0304047); N. Manojlovic, G.A. Mena Marugán, Class.Quant.Grav. 182065 (2001), (preprint gr-qc/0011080); G.A. Mena Marugán Phys.Rev.D 63024005 (2001), (preprint gr-qc/0011068); G.A. Mena Marugan and M. Montejo, Phys.Rev.D 58104017 (1998), (preprint gr-qc/9806105); Phys.Rev.D 61084019 (2000), (preprint gr-qc/9906101).

[9] K.V. Kuchař, in Highlights in gravitation and cosmology, edited by B.R.Iyer, A. Kembhavi, J.V. Narlikar and C.V. Vishveshwara (Cambridge University Press: Cambridge, 1987).

[10] C.G.Torre and M. Varadarajan , Phys. Rev. D 58064007 (1998), (preprint hep-th/9707221).

[11] C.G. Torre and M. Varadarajan, Class. Quantum Grav. 162651 (1999), (preprint hep-th/9811222).

[12] K.V. Kuchař, Phys. Rev. D 392263 (1989).

[13] D. Shale, Trans. Am. Math. Soc. 103149 (1962).

[14] R.M. Wald, Ann. Phys.(N. Y.) 118490 (1979).

[15] N.D. Birrell and P.C.W. Davies, Quantum fields in curved spacetime (Cambridge University Press: Cambridge, 1982).

[16] J.D. Romano and C.G. Torre, Phys. Rev. D 535634 (1996), (preprint gr-qc/9910043).

[17] M. Reed and B. Simon, Methods of Modern Mathematical Physics vol. 1 (New York: Academic, 1972).

[18] A. Corichi, J. Cortez and G.A. Mena Marugán, Phys. Rev. D 73041502 (2006), (preprint gr-qc/0510109); Phys. Rev. D 73084020 (2006), (preprint gr-qc/0603006).

[19] M. Pierri, Int.J.Mod.Phys.D 11135 (2002), (preprint gr-qc/0101013).

[20] A.Corichi, J. Cortez, and H. Quevedo, Int.J.Mod.Phys.D 111451 (2002), (preprint gr-qc/0204053).

[21] C.G. Torre, Phys. Rev. D 66084017 (2002), (preprint gr-qc/0206083).

[22] C. Rovelli, Living Rev. Relativity 1 1998-1 (1998) http://relativity.livingreviews.org/Articles/ lrr-1998-1/index.html.

[23] M. Varadarajan, preprint gr-qc/0607068 (2006).

[24] G.N. Watson A Treatise on the Theory of Bessel Functions (Cambridge University Press: Cambridge, 1966). 